\title{
Matching with Frictions and Entry with Poisson Distributed Buyers and Sellers.*
}

\author{
Peter Norman ${ }^{\dagger}$
}

September 26, 2016.

\begin{abstract}
I consider a directed search model with population uncertainty. Buyers and sellers are randomly drawn from independent Poisson distributions. This provides a simple justification for the usual equilibrium selection where only symmetric randomizations by buyers are considered, because any equilibrium is payoff equivalent to the symmetric continuation equilibrium. The Poisson assumption also makes the model more tractable, making it possible to handle entry, which is difficult with a fixed finite set of sellers. I also demonstrate that the Poisson model belongs to a more general class of models in which prices are strategic complements and equilibria are unique.
\end{abstract}

Keywords: Poisson Distribution, Directed Search, Entry, Supermodularity.

JEL Classification Number: D43, L13.

*I thank Fernando Alvarez, Garth Baughman, Gary Biglaiser, Xinquan Chen, Benoit Julien, John Kennes, Ian King, Philipp Kircher, Ben Lester, Fei Li, Andy McLennan, Claudio Mezzetti, Roger Myerson, Vendela Norman, Can Tian, Ronald Wolthoff, Randy Wright, seminar participants at the Chicago Fed, the Helsinki Center of Economic Research, University of Cologne, and participants at the 2013 Midwest Macro conference at UIUC,the 5th NYU Search Theory workshop, the 2015 Second African Conference on Search and Matching in Marrakesh, and the 2016 Mad Money Conference in Madison, Wisconsin, for comments and discussions. The usual disclaimer applies.

${ }^{\dagger}$ Department of Economics, University of North Carolina at Chapel Hill, 107 Gardner Hall, CB 3305, Chapel Hill, NC 27599-3305. Email: normanp@email.unc.edu 


\section{Introduction}

In models of directed search there are no search frictions in the traditional sense. Instead, the frictions arise because there is a chance that more buyers show up at a given location than the seller has the capacity to serve. Buyers therefore trade off the price with the probability of trading when deciding which seller to visit, which generates some market power for the sellers. However, if $n$ buyers observe prices posted by $m$ different sellers a coordination problem is created for the buyers and unless one seller posts a deal that is so much better than that of the the other sellers that all buyers visit the same seller, there is multiplicity of continuation equilibria. That is, with two buyers and two sellers posting prices not too far apart, buyer 1 may visit seller 1(2) for sure and buyer 2(1) may visit seller 2 for sure, or both buyers may randomize. As shown in Burdett et al [2] this makes it possible to support a continuum of prices in equilibrium.

To get predictive power the literature that follows focuses on equilibria where all buyers follow the same continuation strategy after any posted prices (which is inefficient). This is usually justified with informal arguments suggesting that coordination is difficult, in particular in large markets, but a more formal justification can be found in Bland and Loertscher [1].

The innovation of this paper is to introduce population uncertainty into an otherwise standard directed search model. ${ }^{1}$ That is, it is assumed that there is a set of types of sellers $T$ and a set of types of buyers $C$. Population uncertainty means that the number of any type buyer or seller is a random variable. In standard game theory beliefs are formulated as a distinct randomization for each player. This cannot be the case with population uncertainty, as players cannot be aware of each other's identities. Instead, players are aware of the possible types and their corresponding probability distributions. It cannot be perceived that two individuals of the same type behave differently as others cannot distinguish them, which implies that all players of the same type must randomize in the same way over the available actions. Indeed, this is the reason for introducing $T$ and $C$ in the first place. Without these type spaces, which are assumed payoff irrelevant in this paper, symmetry would be an assumption, whereas non-trivial type spaces allow for coordination where, say, blue buyers go to blue sellers and red buyers go to red sellers.

The tractability of the model considered in this paper comes from assuming that the number of each type of buyer and seller are draws from independent Poisson distributions. As discussed in Myerson [19], [20] the Poisson distribution has a number of unique properties. It satisfies the aggregation property, meaning that sums of independent Poisson distributions are distributed Poisson. It also satisfies the decomposition property, which says that if the number of players of type $t$ is Poisson with parameter $\lambda$ and that players of type $t$ pick some action with probability $p$, then the number of type $t$ players picking the action is a Poisson with parameter $\lambda p$. Finally, the Poisson distribution generates environmental equivalence, implying that players have the same beliefs over the distribution of others as the ex ante beliefs of an outside observer. These properties are all unique to the Poisson distribution, but if players are generated as the sum of many independent random variables the result is approximately Poisson, so the Poisson may be a good approximation

\footnotetext{
${ }^{1}$ See Myerson [19] for details on how to define population uncertainty.
} 
in many circumstances.

The most important result of the paper comes more or less directly from these three properties of the Poisson distribution. I show that it is without loss of generality to focus on equilibria where every type of buyer follows the same strategy. There are also more "coordinated" continuation equilibria where some type buyers are more likely to visit certain sellers than others, but all such continuation equilibria are payoff equivalent to the one where every type buyer follows the same randomization. Because of this payoff equivalence it is now impossible to construct equilibria that punishes or rewards sellers by selecting different types of continuation equilibria depending on which prices are posted. The indeterminacy result in Burdett et al [2] therefore disappears. As far as I know there are no results along these lines in either the directed search literature or the existing literature on Poisson games.

While the most important contribution is to justify the use of symmetric randomizations, the setup with Poisson distributed players also improve tractability relative to the case with a known set of players, much like in the literature on strategic voting. In some sense, it is a compromise between the standard market utility models, where agents live in a continuum, and standard finite models. ${ }^{2}$ Market utility models are usually tractable because no decision of any agent can change the utility of other players, but this comes at the cost of making it conceptually awkward to ask what happens if a single agent deviates from the equilibrium path. To build in sequential rationality it is therefore necessary to use various tricks, such as asking what would happen if a small measure of firms were to deviate and then take the limit as this measure approaches zero. This has led some researchers to ask what the game theoretic foundations of the market utility approach, as seen in Peters [21], [22], Julien et al [10], Burdett et al [2] , Lester [12], and Galenianos and Kircher [5] and others. I believe the setup with Poisson distributed players could be very useful in pushing this frontier further because of its increased tractability. In this paper, I show that an otherwise rather intractable entry problem can be dealt with, and I suspect that there are many other applications.

Naively, the problem of handling entry with a finite set of agents may appear rather uninteresting. One may think that an indifference condition from a continuum model could be replaced by a condition that says that the entry cost must be in between the equilibrium seller profit and the hypothetical equilibrium profit in case an extra seller enters. This is not always the case. The reason is that the profit that a firm makes when $m$ other firms enter is higher when the firm enters the market unexpectedly compared to the case when the other $m$ firms expect the firm to enter. ${ }^{3}$ For this reason, equilibria with pure entry strategies generally fail to exist and mixed strategies are difficult to deal with. In contrast, an essentially unique equilibrium is remarkably simple to characterize with Poisson distributed sellers. In equilibrium, all sellers that enter the market post the same price. The price is strictly decreasing in the entry probability, and the equilibrium is essentially unique in that the average entry probability is determined uniquely by a zero profit condition. Taking the limit as the number of buyers and sellers goes to infinity, the equilibrium

\footnotetext{
${ }^{2}$ Market utility models assume that each participant optimizes given some measure of aggregate market conditions as opposed to explicitly strategic models. See Montgomery [?] and Moen [?]

${ }^{3}$ The only paper I am aware of that deals with this is Geromichalos [6].
} 
approaches the market utility benchmark.

A more or less direct consequence of using the Poisson distribution is that the (expected) matching function delivered from the equilibrium analysis satisfies constant returns to scale. In contrast, the matching function generated from a model with $m$ sellers and $n$ buyers exhibits decreasing returns to scale, although it converges to a constant returns function as $m$ and $n$ tend to infinity. This has interesting implications for the model with entry. It has been shown by Levin and Smith [14] that coordination losses increase with the number of potential entrants, even when actual entry stays the same. In contrast, the equilibrium in this paper's model with entry is invariant to the expected number of sellers as long as not every potential seller enters for sure.

The paper also provides a considerable generalization of results found in Galenianos and Kircher [5] and Kim and Camera [11]. These papers follow the standard approach and assume a symmetric randomization, but allow for a more flexible meeting technology than that in the applied literature. In the Poisson setup, the symmetric randomization is a result, but otherwise it fits into the structure of these papers. I improve upon these papers in two ways. Firstly, I demonstrate that the profit function for a seller is strictly concave in the relevant range without using differentiability, which is also an expositional improvement as the proof is more instructive. Secondly, I show that the reduced form game generated from the sequentially rational (symmetric) randomizations is a supermodular game under somewhat more general conditions than those considered in Galenianos and Kircher [5]. This is useful because supermodular games have a smallest and largest equilibrium, so existence of pure strategy equilibria is immediate.

Additionally, profit functions are strictly concave when restricting the domain to prices in between the smallest and largest equilibria mixed strategy equilibria can be ruled out. I also demonstrate that equilibria are unique under conditions that are almost the same as in Galenianos and Kircher [5] and Kim and Camera [11], but slightly more general. The main value of this result may be the methodology. After ruling out mixed strategies and showing concavity of the profit function, the proof of uniqueness turns out to be a simple inspection of a first order condition that must hold in equilibrium. Finally, the supermodularity allows a test for uniqueness also when the profit function fails to be concave in the range where buyers visit with positive probability: if one (say, by direct computation) can show that there is a unique symmetric equilibrium, then this is also the unique equilibrium of the model (also allowing for mixed strategies). Hence, this result expands the set of environments with unique equilibria (within the class where buyers follow symmetric continuation strategies) relative Kim and Camera [11] and provides a simple test that can be useful in applications.

\section{The Model}

The model is a variant of Burdett et al. [2] where buyers and sellers are drawn from a Poisson distribution, and where sellers face a non-trivial entry decision. Each seller is endowed with an indivisible object she attaches no value to, but that all buyers value at 1 . 
1. First, nature draws $s$ potential sellers and $b$ buyers. Players do not have unique names, but I allow for observable payoff irrelevant characteristics that potentially can be used as coordinating devices. Formally, let $t \in T=\{1, \ldots, T\}$ be a generic seller type and $c \in C=$ $\{1, \ldots, C\}$ be a generic buyer type. It is assumed that the number of type $t$ sellers is a draw from a Poisson with expected value $m_{t}$ and the number of type $c$ buyers are drawn from a Poisson with expected value $n_{c}$. The number of type $t(c)$ sellers (buyers) is independent from the number of $t^{\prime}$ sellers or $c^{\prime}$ buyers for any $\left(t, t^{\prime}, c^{\prime}\right)$ or $\left(c, t^{\prime}, c^{\prime}\right)$. As adding two independent Poisson distributions generates a new Poisson distribution with an expectation equal to the sum of the expectations we have that $m=\sum_{t \in T} m_{t}$ is the expected number of potential sellers in the market and $n=\sum_{c \in C} n_{c}$ is the expected number of buyers.

2. Each potential seller decides whether or not to enter the market. Conditional on entry sellers post prices. Entry and price posting is assumed to be simultaneous, so sellers cannot condition prices on the number of other sellers or the distribution of types. Additionally, the realized number and distribution of types for the buyers is assumed to be unobservable to all players, and therefore noncontractable. A seller that enters pays utility cost $k \in(0,1)$.

3. Buyers that are "born" observe how many sellers of each type are in the market and the price posted by each seller. A randomized strategy for a buyer is thus a probability distribution over which seller to visit, which is allowed to depend the identity of the seller (not just the seller type) and the type of the buyer. ${ }^{4}$

4. If at least one buyer visits a seller one of the visiting buyers is chosen randomly to receive the good at the posted price.

\section{The Poisson Assumption Justifies Lack of Coordination}

In directed search models with a finite set of agents there is a plethora of equilibria that can be supported by buyers coordinating on qualitatively different continuation equilibria after different price vectors posted by the sellers. Almost all of the literature, however, focuses on the case where buyers play a symmetric mixed strategy after any history of prices posted. This approach is typically justified by arguing that coordination is hard in large anonymous markets. An important contribution of this paper is that having players being randomly drawn from a Poisson distribution justifies using symmetric mixed strategies for the buyers.

\footnotetext{
${ }^{4}$ The asymmetry between buyers and sellers at this stage occurs because, following standard assumptions in the directed search literature, the buyers observe all sellers, their types, and their prices. Hence, sellers have ex post "identities". In contrast, buyers' choices are made under the veil of ignorance about the realized number and buyer types.
} 


\subsection{Strategies and Equilibrium}

A pure strategy of a seller is a type specific price and entry decision, that is, a map $\xi: T \rightarrow$ $\{0,1\} \times R_{+}$where $\xi_{1}(t)=1$ means that the seller enters and $\xi_{2}(t)$ is the price posted by a type $t$ seller. It will be shown later on that no equilibria exist where sellers randomize over prices, whereas mixed strategies will be useful to deal with the entry decisions.

All buyers observe the types of the sellers that have entered the market and the price of each entrant. Hence, a typical history observed by a buyer is a vector $\left(\left(p_{1}, t_{1}\right), \ldots .,\left(p_{s}, t_{s}\right)\right)$ where $1, \ldots ., s$ are (ex post) labels for the entering sellers and $t_{j}$ is the type of seller $j=1, \ldots, s$. We notice that the seller type is redundant as it is irrelevant whether the index $j$ is a number-type pair or just a number. Hence, we may simplify notation slightly and describe a buyer continuation strategy as a map $\theta: C \times R_{+}^{s} \rightarrow \Delta^{s}$, where we write $p^{s}=\left(p_{1}, \ldots, p_{s}\right)$ for a generic configuration of prices when $s$ sellers is in the market and where $\theta_{j}\left(c, p^{s}\right)$ denotes the probability that a type $c$ buyer visits seller $j$ when $p^{s}$ are the posted prices.

In order to write down the appropriate payoff functions we first need to appeal to some properties of the Poisson distribution. ${ }^{5}$ Firstly, as mentioned above, it satisfies the aggregation property, meaning that a sum of independent Poisson random variables with parameters $\left(\lambda_{1}, \ldots ., \lambda_{r}\right)$ is distributed Poisson with parameter $\sum_{j=1}^{r} \lambda_{j}$. This is seen by noting that for any positive $k$ we have that

$\operatorname{Pr}\left[x_{1}+x_{2}=k\right]=\sum_{v=0}^{k} \frac{e^{-\lambda_{1}} \lambda_{1}^{v}}{v !} \frac{e^{-\lambda_{2}} \lambda_{2}^{k-v}}{(k-v) !}=\frac{e^{-\left(\lambda_{1}+\lambda_{2}\right)}}{k !} \sum_{v=0}^{k} \frac{k !}{v !(k-v) !} \lambda_{1}^{v} \lambda_{2}^{k-v}=\frac{e^{-\left(\lambda_{1}+\lambda_{2}\right)}}{k !}\left(\lambda_{1}+\lambda_{2}\right)^{v}$,

where the first equality uses the convolution formula for discrete random variables and the fact that $\frac{e^{-\lambda_{i}} \lambda_{i}^{k}}{k !}$ is the probability of $x_{i}=k$, the second equality is algebra, and the final equality is the binomial expansion.

Somewhat less known is what Myerson [19] refers to as the decomposition property of the Poisson distribution. To explain this property it is useful to phrase it in terms of population uncertainty, so that the number of agents is a Poisson with parameter $\lambda$. Also, suppose that each agent in the population is randomly assigned some characteristic with probability $\theta$. Then, the probability distribution over the number of agents with the characteristic is a Poisson with parameter $\lambda \theta$. This follows because for any $k=0,1$...the probability that $k$ agents have the characteristic conditional on there being $v$ agents comes from a binomial with parameters $\theta$ and $v$. The unconditional probability is thus,

$$
\begin{aligned}
\sum_{v=k}^{\infty} \frac{e^{-\lambda} \lambda^{v}}{v !} \frac{v !}{k !(v-k) !} \theta^{k}(1-\theta)^{v-k} & =\frac{e^{-\lambda}(\theta \lambda)^{k}}{k !} \sum_{v=k}^{\infty} \frac{1}{(v-k) !}[\lambda(1-\theta)]^{v-k} \\
& =\frac{e^{-\lambda}(\theta \lambda)^{k}}{k !} \sum_{r=0}^{\infty} \frac{1}{r !}[\lambda(1-\theta)]^{r} \\
& =\frac{e^{-\lambda}(\theta \lambda)^{k}}{k !} e^{\lambda(1-\theta)}=\frac{e^{-\lambda \theta}(\theta \lambda)^{k}}{k !}
\end{aligned}
$$

${ }^{5}$ The reader may consult Haight [8] for further details. 
Finally, the Poisson distribution satisfies what Myerson [19] calls environmental equivalence. This means that if the number of players is a Poisson with mean $\lambda$, then the probability distribution over the number of players for an outside observer is the same as the probability distribution over the number of other players for a player (of any type) in the game. In other words, being present in the game provides no information about the distribution of the other players. No other probability distribution satisfies this property. ${ }^{6}$

To see how this is a remarkable property, note that there are two competing effects at play. Being a player is evidence in favor of there being more players than initially thought. In addition, being a player is also evidence in favor of there being fewer players other than oneself. The remarkable consequence of the Poisson assumption is that these two effects exactly cancel each other out.

\subsubsection{Buyer Optimality Conditions}

Now, consider a history summarized (without loss of generality) by price vector $p^{s}=\left(p_{1}, \ldots, p_{s}\right)$ and consider an arbitrary buyer continuation $\theta: C \times R_{+}^{s} \rightarrow \Delta^{s}$. By the decomposition property we have that arrivals of type $c$ buyers at seller with ex post index $j$ is a Poisson with mean $\theta_{j}\left(c, p^{s}\right) n_{c}$. By the aggregation property the total number of buyers arriving at $j$ is a Poisson with mean $\sum_{c \in C} \theta_{j}\left(c, p^{s}\right) n_{c}$ and by environmental equivalence this Poisson is also the perceived distribution over total arrivals at $j$ for any buyer of any type. Given that sellers treat buyers equally when more than one buyer arrives, the probability of trading for a buyer who visits $j$ is

$$
\begin{aligned}
\operatorname{Pr} \text { [trading with seller } j] & =\sum_{v=0}^{\infty} \frac{1}{v+1} \underbrace{\frac{e^{-\sum_{c \in C} \theta_{j}\left(c, p^{s}\right) n_{c}}}{v !}\left(\sum_{c \in C} \theta_{j}\left(c, p^{s}\right) n_{c}\right)^{v}}_{\text {Prob } v \text { other buyers }} \\
& =\frac{1}{n \theta_{i}} \sum_{v=0}^{\infty} \frac{e^{-\sum_{c \in C} \theta_{j}\left(c, p^{s}\right) n_{c}}}{(v+1) !}\left[\sum_{c \in C} \theta_{j}\left(c, p^{s}\right) n_{c}\right]^{v+1} \\
& =\frac{1}{\sum_{c \in C} \theta_{j}\left(c, p^{s}\right) n_{c}}\left[\sum_{v=0}^{\infty} \frac{e^{-\sum_{c \in C} \theta_{j}\left(c, p^{s}\right) n_{c}}}{v !}\left[\sum_{c \in C} \theta_{j}\left(c, p^{s}\right) n_{c}\right]^{v}-\frac{e^{-\sum_{c \in C} \theta_{j}\left(c, p^{s}\right) n_{c}}}{0 !}\right] \\
& =\frac{\left(1-e^{-\sum_{c \in C} \theta_{j}\left(c, p^{s}\right) n_{c}}\right.}{\sum_{c \in C} \theta_{j}\left(c, p^{s}\right) n_{c}}
\end{aligned}
$$

Moreover, again from the environmental equivalence property of the Poisson, the sellers expect arrivals in accordance with the same Poisson distributions that buyers use for arrivals of other buyers, so the expected payoff for seller (with ex post index) $j$ is $^{7}$

$$
p_{j}\left(1-e^{-\left[\sum_{c \in C} \theta_{j}\left(c, p^{s}\right) n_{c}\right]}\right) .
$$

We can now formally define a continuation equilibrium:

${ }^{6}$ See Theorem 2 in Myerson [19].

${ }^{7}$ Environmental equivalence also plays a role in Lester [13] where it is called independence. 
Definition 1 The buyer visitation strategy $\theta: C \times R_{+}^{s} \rightarrow \Delta^{s}$ is a continuation equilibrium strategy if

$$
\left(1-p_{j}\right) \frac{\left(1-e^{-\left[\sum_{c \in C} \theta_{j}\left(c, p^{s}\right) n_{c}\right]}\right)}{\sum_{c \in C} \theta_{j}\left(c, p^{s}\right) n_{c}}=\left(1-p_{i}\right) \frac{\left(1-e^{-\left[\sum_{c \in C} \theta_{i}\left(c, p^{s}\right) n_{c}\right]}\right)}{\sum_{c \in C} \theta_{i}\left(c, p^{s}\right) n_{c}}
$$

for each pair $(i, j)$ such that there exists $c, c^{\prime}$ with $\theta_{i}\left(c, p^{s}\right)>0$ and $\theta_{j}\left(c^{\prime}, p^{s}\right)>0$ and if

$$
\left(1-p_{j}\right) \frac{\left(1-e^{-\left[\sum_{c \in C} \theta_{j}\left(c, p^{s}\right) n_{c}\right]}\right)}{\sum_{c \in C} \theta_{j}\left(c, p^{s}\right) n_{c}} \geq\left(1-p_{i}\right)
$$

if $\theta_{i}\left(c, p^{s}\right)=0$ for every $c \in C$ and $\theta_{j}\left(c^{\prime}, p^{s}\right)>0$ for some $c^{\prime} \in C$

Given this characterization of the continuation equilibrium payoffs our first result is almost immediate.

Proposition 1 Suppose that $\theta: C \times R_{+}^{s} \rightarrow \Delta^{s}$ is a continuation equilibrium buyer strategy. Then there exists a symmetric equilibrium continuation strategy $\widehat{\theta}: C \times R_{+}^{s} \rightarrow \Delta^{s}$ (satisfying $\widehat{\theta}_{j}\left(c, p^{s}\right)=$ $\widehat{\theta}_{j}\left(c^{\prime}, p^{s}\right)$ for every $s, p^{s}, j \in\{1, \ldots, s\}$ and every $\left.\left(c, c^{\prime}\right) \in C\right)$ such that every buyer and every seller earns the same payoff when buyers use $\widehat{\theta}$ as when buyers use $\theta$.

Proof. For any $s, p^{s}, j \in\{1, \ldots, s\}$, and $c \in C$ consider the randomization probability

$$
\widehat{\theta}_{j}\left(c, p^{s}\right)=\frac{\sum_{c \in C} \theta_{j}\left(c, p^{s}\right) n_{c}}{\sum_{c \in C} n_{c}}
$$

which (again using the decomposition and aggregation properties) generates arrival rate

$$
\sum_{c \in C} \widehat{\theta}_{j}\left(c, p^{s}\right) n_{c}=\sum_{c \in C} \widehat{\theta}_{j}\left(c, p^{s}\right)\left[\frac{\sum_{c \in C} \theta_{j}\left(c, p^{s}\right) n_{c}}{\sum_{c \in C} n_{c}}\right]=\sum_{c \in C} \theta_{j}\left(c, p^{s}\right) n_{c}
$$

Hence, the seller payoff in (4) is unchanged as are the buyer payoffs in (5) and (6). Thus, $\widehat{\theta}$ : $C \times R_{+}^{s} \rightarrow \Delta^{s}$ is a continuation equilibrium provided that $\theta: C \times R_{+}^{s} \rightarrow \Delta^{s}$ is a continuation equilibrium.

Therefore, neither buyers nor sellers can do any better or worse by conditioning on payoff irrelevant buyer characteristics. In the remainder of the paper I therefore assume that the buyers follow a symmetric mixed strategy following any posted prices, which is without loss of generality in terms of equilibrium payoffs. Note the contrast between this result and the characterization of continuation equilibria in Peters [21], Burdett et al [2] and other directed search papers with a fixed finite set of players. In these papers there is always multiplicity of equilibria following histories where a randomized continuation equilibrium exists, and the equilibria are not payoff equivalent because they differ in degree of coordination. As pointed out in Burdett et al [2] this implies that a continuum of price vectors can be supported as equilibria by having the buyers punishing and rewarding the sellers by coordinating on qualitatively different continuation equilibria following different price vectors. In contrast, in the current model such punishments and rewards are 
inconsistent with sequential rationality, and we can, without loss of generality, focus on equilibria where all buyers follow the same continuation strategy after any history.

For simplicity of notation we define $G(\theta)=\left(1-e^{-\theta n}\right) / \theta n$ and, abusing previous notation, we now write $\theta: R_{+}^{s} \rightarrow \Delta^{s}$ and $\theta_{j}\left(p^{s}\right)$ for a buyer symmetric visitation strategy. The conditions for a buyer symmetric continuation equilibrium are then that

$$
\left(1-p_{j}\right) G\left(\theta_{j}\left(p^{s}\right)\right)=\left(1-p_{i}\right) G\left(\theta_{i}\left(p^{s}\right)\right)
$$

holds if $\theta_{j}\left(p^{s}\right)>0$ and $\theta_{i}\left(p^{s}\right)>0$ and that $\left(1-p_{j}\right) G\left(\theta_{j}\left(p^{s}\right)\right) \geq\left(1-p_{i}\right) G(0)=\left(1-p_{i}\right)$ if $\theta_{j}\left(p^{s}\right)>0$ and $\theta_{i}\left(p^{s}\right)=0$.

\subsubsection{Generalizing the Non-Coordination Result}

For ease of notation, Proposition 1 is stated and proved in the context of the simple setup with representative buyers and sellers that will be considered for the remainder of the paper. However, it should be clear that it is much more general. Consider the case with "principals" posting some contracts (having the role of the sellers above) and agents responding to these contracts by randomizing over where to visit. Provided that rationing can only depend on payoff relevant agent characteristics (such as productive versus unproductive workers), it is just a matter of creating more notation to show that it is without loss to assume that agents with no payoff relevant differences will visit any given principal with the same probability. Qualitatively, the argument is identical.

\subsubsection{Seller Optimality Conditions}

An equilibrium consists of a type dependent entry strategy $\boldsymbol{\alpha}^{*}=\left(\alpha_{1}^{*}, \ldots, \alpha_{T}^{*}\right)$ and a price vector $\boldsymbol{p}^{*}=\left(p_{1}^{*}, \ldots, p_{T}^{*}\right)$ that is optimal given the buyer continuation equilibrium strategies. In contrast to buyers, sellers face uncertainty both with the respect of the number of buyers and sellers, which makes it necessary to keep track of the number of entering sellers of each type in order to formulate the expected profit function for a seller.

To accomplish this, consider the problem for a seller. Let $I=\left(I_{1}, \ldots, I_{T}\right)$ be a generic ex post "entry vector" so that $I_{t}$ denotes the number of type $t$ sellers (other than the seller under consideration if of the same type). We then know from the analysis of the buyer continuation strategies that if the seller enters posting a price $p$, then the probability of a visit depends only on the price and not on the type as otherwise the conditions in (9) cannot hold. We can therefore write $\theta\left(p, \boldsymbol{p}^{*}, I\right)$ for the probability that a buyer visits a seller posting price $p$ provided that other sellers stick to the hypothetical equilibrium price vector $\boldsymbol{p}^{*}=\left(p_{1}^{*}, \ldots, p_{T}^{*}\right)$ and that the entry of other types is described by $I=\left(I_{1}, \ldots, I_{T}\right) \in N^{T}$.

By the Poisson decomposition property, if type $t$ enters with probability $\alpha_{t}$ the number of type $t$ sellers in the market is Poisson distributed with expectation $\alpha_{t} m_{t}$ and by environmental equivalence this is also the perceived distribution over other sellers entering for any individual 
seller contemplating entry, so the net expected profit of a seller that enters can be written as

$$
\pi\left(p, \mathbf{p}^{*}, \boldsymbol{\alpha}^{*}\right)=\left[\sum_{I_{1}=0}^{\infty} \ldots . . \sum_{I_{T}=0}^{\infty}\right] \prod_{t=1}^{T} \frac{e^{-\left(\alpha_{t}^{*} m_{t}\right)}\left(\alpha_{t}^{*} m_{t}\right)^{I_{t}}}{I_{t} !} p\left(1-e^{-\theta\left(p, \mathbf{p}^{*}, I\right) n}\right)-K .
$$

What is remarkable about (10) is that all potential entrants face the same optimization problem for every entry strategy $\boldsymbol{\alpha}^{*}=\left(\alpha_{1}^{*}, \ldots, \alpha_{T}^{*}\right)$ and price vector $\boldsymbol{p}^{*}=\left(p_{1}^{*}, \ldots, p_{T}^{*}\right)$. That is, symmetric models often have symmetric equilibria, but in models with role identification, symmetry is lost outside the equilibrium, while in the current model with random sellers even the best responses are symmetric. Hence, if we can argue that best responses are unique, it is immediate that any equilibrium is symmetric, and if we in addition can guarantee the profit to be concave, then the equilibrium is unique. Thus, it is necessary to investigate the properties of $p\left(1-e^{-\theta\left(p, \mathbf{p}^{*}, I\right) n}\right)$, which is done in the next section.

\section{General Properties of The Demand System in Directed Search}

For the purposes of this section, I will treat $G(\cdot)$ as a generic matching probability for the buyer as a function of the probability that a buyer visits the seller. Leading examples are $G(\theta)=$ $\left(1-e^{-\theta n}\right) / \theta n$, the main case in this paper, and $G(\theta)=1-(1-\theta)^{n} / n \theta$ as in Burdett et al [2] and most other papers considering finite models, but there are many other possibilities (see Galenianos and Kircher [5] for a number of other examples). As the number of active sellers is kept fixed through this analysis we abuse previous notation and write $\boldsymbol{p}$ for a generic price vector. We note that a requirement for buyer and seller matching probabilities to be consistent is that

$$
\operatorname{Pr}[\text { seller } j \text { trades }]=n \operatorname{Pr}[\text { buyer tradesl } \mid \text { visit } j] \times \operatorname{Pr}[\text { buyer visits } j]=n \theta_{j}(\boldsymbol{p}) G\left(\theta_{j}(\boldsymbol{p})\right),
$$

where in equilibrium $\left\{\theta_{j}(\boldsymbol{p})\right\}_{j=1}^{s}$ satisfies $\sum_{j=1}^{s} \theta_{j}(\boldsymbol{p})=1$ and $0 \leq \theta_{j}(\boldsymbol{p}) \leq 1$ for each $j$ and solves

$$
\left(1-p_{j}\right) G\left(\theta_{j}(\boldsymbol{p})\right)=\left(1-p_{i}\right) G\left(\theta_{i}(\boldsymbol{p})\right)
$$

for $\theta_{j}(\boldsymbol{p})>0$, and $\theta_{i}(\boldsymbol{p})>0$ and

$$
\left(1-p_{j}\right) G\left(\theta_{j}(\boldsymbol{p})\right) \geq\left(1-p_{i}\right) G(0)
$$

if $\theta_{j}(\boldsymbol{p})>0$, and $\theta_{i}(\boldsymbol{p})=0$. We first establish that there is a unique solution to the demand system defined by (12) and (13) for any reasonable choice of $G$ :

Proposition 2 Suppose that $G$ is continuous and strictly decreasing and fix any $\boldsymbol{p}$. Then, the system of randomization probabilities defined by the equalities in (12) and inequalities in (13) has a unique solution $\left\{\theta_{j}(\boldsymbol{p})\right\}_{j=1}^{s}$ Moreover;

1. $\theta_{j}(\boldsymbol{p})$ is decreasing in $p$

2. $\theta_{j}(\boldsymbol{p})$ is increasing in $p_{i}$ for any $i \neq j$ 
3. $\theta_{j}(\boldsymbol{p})$ is strictly decreasing in $p_{j}$ in the range where $0<\theta_{j}(\boldsymbol{p})<1$

4. $\theta_{j}(\boldsymbol{p})$ is strictly increasing in $p_{i} \neq p_{j}$ in the range where $0<\theta_{i}(\boldsymbol{p})<1$.

A proof is in the appendix, where the case with corners is dealt with carefully. The idea is rather simple though, and may be understood from considering the case in which prices are such that all sellers are visited with positive probability. First, suppose there is non-uniqueness. Then, the probability of a visit must be strictly larger at one seller and strictly lower at some other seller when comparing two equilibria. Thus, buyers are strictly worse off visiting one seller in the first equilibrium than in the second equilibrium, and buyers are strictly better off visiting some other seller in the first equilibrium. Obviously, this is inconsistent with the indifference conditions in (12) being satisfies in both equilibria. Extending the argument to deal with the case when not all sellers are visited requires a little bit of work, but the key idea is unchanged as the non-uniqueness implies that all buyers strictly prefer one continuation equilibrium to the other, which is impossible. This monotonicity is also what underlies the monotone comparative statics results in Proposition 2.

Hence, we now know that if there is a given set of sellers $j=\{1, \ldots, s\}$, then the profit for each seller given any $\boldsymbol{p}$ is determined as the unique solution $\left\{\theta_{j}(\boldsymbol{p})\right\}_{j=1}^{s}$ to (12) and (13). Additionally, (11) implies that the profit function in this more general case (still assuming that the set of sellers is given) is of the form

$$
\pi_{j}(\boldsymbol{p})=h_{j}\left(p_{j}\right) n \theta_{j}(\boldsymbol{p}) G\left(\theta_{j}(\boldsymbol{p})\right),
$$

where we have also generalized the profit relative to basic symmetric seller setup by assuming that the profit conditional on a successful match is $h_{j}\left(p_{j}\right)$ rather than $p_{j}$. This allows for different sellers having different unit costs and various other generalizations in which sellers are offering one-dimensional utility promises to buyers.

Our next task is to argue that the profit function is concave. The main difficulty is to guarantee that the visitation probability is concave, which I do as a separate Lemma:

Lemma 1 Suppose that $G$ is strictly decreasing and convex and that $1 / G$ is strictly convex. Then, $\theta_{j}(\boldsymbol{p})$ is strictly concave in $p_{j}$ in range where $0<\theta_{j}(\boldsymbol{p})<1$.

This is a slight generalization of Galenianos and Kircher [5], where the difference is that Lemma 1 doesn't rely on differentiability. Although this difference may not seem important, the logic of the proof in the appendix is arguably considerably easier to follow.

With Lemma 1 in hand it is relatively easy to come up with an additional (and very natural) condition that guarantees that the profit in (14) is concave in $p_{j}$.

Proposition 3 Suppose that $G$ is strictly decreasing and convex and that $1 / G$ is strictly convex and that the product $\theta G(\theta)$ is increasing in $\theta$. Furthermore assume that $h_{j}$ is positive valued, concave and increasing. Then, $\theta_{j}(\boldsymbol{p}) G\left(\theta_{j}(\boldsymbol{p})\right)$ and therefore also the profit function $\pi_{j}(\boldsymbol{p})=$ $h_{j}\left(p_{j}\right) n \theta_{j}(\boldsymbol{p}) G\left(\theta_{j}(\boldsymbol{p})\right)$ is strictly concave in $p_{j}$ over the range where $0<\theta_{j}(\mathbf{p})<1$ 
The added condition that $\theta G(\theta)$ is increasing in $\theta$ is not implied by the other restrictions on $G$, but it is a rather weak condition to impose on the matching technology as the interpretation is that the probability that the seller trades is strictly increasing in the probability that a buyer visits. It appears difficult to come up with examples that one would want to consider that would violate this property. Also note that the restriction that $h_{j}$ is positive valued is without loss of generality as any $p_{j}$ such that $h_{j}\left(p_{j}\right)$ is negative would be strictly dominated. Hence, one may simply restrict the feasible set of prices for the seller to those that generate weakly positive profits if a buyer shows up.

\subsection{The Differentiable Case}

In the case when $G$ is differentiable we have that the indifference conditions (12) imply that

$$
\begin{aligned}
-G\left(\theta_{j}(\boldsymbol{p})\right)+\left(1-p_{j}\right) G^{\prime}\left(\theta_{j}(\boldsymbol{p})\right) \frac{\partial \theta_{j}(\boldsymbol{p})}{\partial p_{j}} & =\left(1-p_{i}\right) G^{\prime}\left(\theta_{i}(\boldsymbol{p})\right) \frac{\partial \theta_{i}(\boldsymbol{p})}{\partial p_{j}} \\
& =\left(1-p_{j}\right) G\left(\theta_{j}(\boldsymbol{p})\right) \frac{G^{\prime}\left(\theta_{i}(\boldsymbol{p})\right)}{G\left(\theta_{i}(\boldsymbol{p})\right)} \frac{\partial \theta_{i}(\boldsymbol{p})}{\partial p_{j}}
\end{aligned}
$$

must hold whenever $(i, j)$ are both visited with positive probability. Summing over $i \neq j$ and rearranging we find that if all sellers are visited with positive probability

$$
\frac{\partial \theta_{j}(\mathbf{p})}{\partial p_{j}}=\frac{G\left(\theta_{j}(\mathbf{p})\right)}{\left(1-p_{j}\right) G^{\prime}\left(\theta_{j}(\mathbf{p})\right)} \frac{\sum_{i \neq j} \frac{G\left(\theta_{i}(\mathbf{p})\right)}{G^{\prime}\left(\theta_{i}(\mathbf{p})\right)}}{\sum_{i=1}^{s} \frac{G\left(\theta_{i}(\mathbf{p})\right)}{G^{\prime}\left(\theta_{i}(\mathbf{p})\right)}} .
$$

Substituting back into (15) we also have that the cross derivatives

$$
\frac{\partial \theta_{i}(\boldsymbol{p})}{\partial p_{j}}=-\frac{G\left(\theta_{j}(\mathbf{p})\right)}{\left(1-p_{j}\right) G^{\prime}\left(\theta_{j}(\mathbf{p})\right)} \frac{\frac{G\left(\theta_{i}(\mathbf{p})\right)}{G^{\prime}\left(\theta_{i}(\mathbf{p})\right)}}{\sum_{i=1}^{s} \frac{G\left(\theta_{i}(\mathbf{p})\right)}{G^{\prime}\left(\theta_{i}(\mathbf{p})\right)}} .
$$

Adding the assumption that $G$ is twice differentiable we can show that the price setting game implied by the demand system defined by (12) and (13) is strictly supermodular. That is, given any $\boldsymbol{p}$ such that $0<\theta_{j}(\boldsymbol{p})<1$ and any $i \neq j$, the cross derivative is strictly positive.

Proposition 4 Suppose that $G$ is twice differentiable, strictly decreasing, convex, $1 / G$ is strictly convex, and that $\theta G(\theta)$ is strictly increasing in $\theta$. Also assume that $h_{j}$ is positive valued and increasing. Then, the profit function defined in (14) satisfies $\frac{\partial^{2} \pi_{j}(\boldsymbol{p})}{\partial p_{i} \partial p_{j}}>0$ over the range where $0<\theta_{j}(\mathbf{p})<1$ ( and $\frac{\partial^{2} \pi_{j}(\boldsymbol{p})}{\partial p_{i} \partial p_{j}} \geq 0$ globally).

As is well known from the literature on monotone comparative statics (see Topkis Milgrom and Shannon [15] and Shannon [23]) a positive cross derivative implies that $\pi_{j}$ satisfies increasing differences, which means that if $p_{j}^{\prime}>p_{j}^{\prime \prime}$ and $\boldsymbol{p}_{-j}^{\prime} \geq \boldsymbol{p}_{-j}^{\prime \prime}$ then

$$
\pi_{j}\left(p_{j}^{\prime}, \boldsymbol{p}_{-j}^{\prime}\right)-\pi_{j}\left(p_{j}^{\prime \prime}, \boldsymbol{p}_{-j}^{\prime}\right) \geq \pi_{j}\left(p_{j}^{\prime}, \boldsymbol{p}_{-j}^{\prime \prime}\right)-\pi_{j}\left(p_{j}^{\prime \prime}, \boldsymbol{p}_{-j}^{\prime \prime}\right)
$$


I suspect that (18) is also true without differentiability of $G$, but the proof of Proposition 4 uses the explicit expressions of the derivatives in (16) and (17) to bound a term involving a cross derivative of $\theta_{j}(\mathbf{p})$. Since $p_{j}$ may without loss be restricted to be in $[0,1]$ for each player, the payoff functions are defined on a compact sub-lattice. Additionally, payoff functions are obviously continuous in all variables, quasi-supermodularity of $\pi_{j}$ in $p_{j}$ holds trivially, and the increasing difference property (18) is a sufficient condition for payoff functions to satisfy single crossing Milgrom and Shannon [15] and Shannon [23]. Hence, we can conclude that the reduced form pricing game is a game with strategic complementarities in the sense of Milgrom and Shannon [15] and by applying Theorem 12 we conclude. ${ }^{89}$

Proposition 5 Suppose that $G$ is twice differentiable, strictly decreasing, convex, that $1 / G$ is strictly convex, $\theta G(\theta)$ is strictly increasing in $\theta$, each $h_{j}$ is increasing and positive valued, and consider the simultaneous move game with payoff functions given by (14). Then, for each $j$ there exists a smallest price $\underline{p}_{j}$ and a largest price $\bar{p}_{j}$ that survive iterated elimination of strictly dominated strategies. Moreover, $\underline{\boldsymbol{p}}=\left(\underline{p}_{1}, \ldots, \underline{p}_{s}\right)$ and $\bar{p}=\left(\bar{p}_{1}, \ldots, \bar{p}_{s}\right)$ are Nash equilibria.

Next, note that if the game is symmetric then the largest and smallest equilibria are necessarily symmetric. Hence, multiplicity can occur only if there are multiple symmetric equilibria. Dropping the firm subscript and writing $h\left(p_{j}\right)$ instead of $h_{j}\left(p_{j}\right)$ and evaluating the first order condition at a symmetric equilibrium $\boldsymbol{p}^{*}=\left(p^{*}, \ldots, p^{*}\right)$ we have that

$$
\begin{aligned}
0 & =h^{\prime}\left(p^{*}\right) \theta_{j}\left(\boldsymbol{p}^{*}\right) G\left(\theta_{j}\left(\boldsymbol{p}^{*}\right)\right)+h\left(p^{*}\right)\left[G\left(\theta_{j}\left(\boldsymbol{p}^{*}\right)\right)+\theta_{j}\left(\boldsymbol{p}^{*}\right) G^{\prime}\left(\theta_{j}\left(\boldsymbol{p}^{*}\right)\right)\right] \frac{\partial \theta_{j}\left(\boldsymbol{p}^{*}\right)}{\partial p_{j}} \\
& =h^{\prime}\left(p^{*}\right) \frac{1}{s} G\left(\frac{1}{s}\right)+h\left(p^{*}\right)\left[G\left(\frac{1}{s}\right)+\frac{1}{s} G^{\prime}\left(\frac{1}{s}\right)\right] \frac{G\left(\frac{1}{s}\right)}{\left(1-p^{*}\right) G^{\prime}\left(\frac{1}{s}\right)} \frac{s-1}{s}
\end{aligned}
$$

after substituting from (16) and evaluating at a symmetric equilibrium. Note that concavity implies that $h^{\prime}(p)$ is decreasing. Moreover, $\frac{h(p)}{1-p}$ is a product of two increasing and (in the relevant range) positive valued functions and therefore increasing, and

$$
\left[G\left(\frac{1}{s}\right)+\frac{1}{s} G^{\prime}\left(\frac{1}{s}\right)\right] \frac{G\left(\frac{1}{s}\right)}{G^{\prime}\left(\frac{1}{s}\right)} \frac{s-1}{s}<0
$$

since $\theta G(\theta)$ is strictly increasing, $G(\theta)>0$, and $G^{\prime}(\theta)<0$. Taken together this implies that the right hand side in (19) is strictly decreasing as a function of $p^{*}$. Under the additional assumption of weak concavity of $h(\cdot)$ we can therefore conclude that equilibria are unique in the case with symmetric sellers.

The same holds with asymmetric sellers, but the proof involves some more work because one needs to deal with the case with $\theta_{j}\left(\boldsymbol{p}^{*}\right) \neq \theta_{j}\left(\mathbf{p}^{* *}\right)$. This is done as follows. Suppose that there are multiple equilibria, then, because of supermodularity there is a smallest equilibrium $\boldsymbol{p}^{*}$ and a

\footnotetext{
${ }^{8}$ See page 175 in Milgrom and Shannon [15]

${ }^{9} \mathrm{I}$ am not the first using techniques from monotone comparative statics in the search literature. See Choi [3]Moscarini and Vinay-Postel, and Gonzalez and Shi. [7].
} 
largest equilibrium $\mathbf{p}^{* *}$, where $p_{j}^{*}<p_{j}^{* *}$ for each seller. Moreover, the first order optimality condition must be satisfied for each seller in each equilibrium, so that

$$
\begin{aligned}
& 0=h_{j}^{\prime}\left(p_{j}^{*}\right) \theta_{j}\left(\boldsymbol{p}^{*}\right) G\left(\theta_{j}\left(\boldsymbol{p}^{*}\right)\right)+h_{j}\left(p_{j}^{*}\right)\left[G\left(\theta_{j}\left(\boldsymbol{p}^{*}\right)\right)+\theta_{j}\left(\boldsymbol{p}^{*}\right) G^{\prime}\left(\theta_{j}\left(\boldsymbol{p}^{*}\right)\right)\right] \frac{\partial \theta_{j}\left(\boldsymbol{p}^{*}\right)}{\partial p_{j}} . \\
& 0=h_{j}^{\prime}\left(p_{j}^{* *}\right) \theta_{j}\left(\boldsymbol{p}^{* *}\right) G\left(\theta_{j}\left(\boldsymbol{p}^{* *}\right)\right)+h_{j}\left(p_{j}^{* *}\right)\left[G\left(\theta_{j}\left(\boldsymbol{p}^{* *}\right)\right)+\theta_{j}\left(\boldsymbol{p}^{* *}\right) G^{\prime}\left(\theta_{j}\left(\boldsymbol{p}^{* *}\right)\right)\right] \frac{\partial \theta_{j}\left(\boldsymbol{p}^{* *}\right)}{\partial p_{j}},
\end{aligned}
$$

where $\frac{\partial \theta_{j}(\boldsymbol{p})}{\partial p_{j}}$ is defined in (16). Inspecting these expressions one notes that the "direct effect" of changing $p_{j}$ (effects that don't work through changes in visitation probabilities) has a monotone effect of the right hand side of the first order condition in (21). Hence, it is impossible to have multiplicity unless the visitation probabilities change for the same reason as in the symmetric model. Moreover, probabilities must add up, so there must be some seller $j$ with $\theta_{j}\left(\boldsymbol{p}^{*}\right)>\theta_{j}\left(\boldsymbol{p}^{* *}\right)$, which immediately implies that

$$
h_{j}^{\prime}\left(p_{j}^{*}\right) \theta_{j}\left(\boldsymbol{p}^{*}\right) G\left(\theta_{j}\left(\boldsymbol{p}^{*}\right)\right)>h_{j}^{\prime}\left(p_{j}^{* *}\right) \theta_{j}\left(\boldsymbol{p}^{* *}\right) G\left(\theta_{j}\left(\boldsymbol{p}^{* *}\right)\right)>0,
$$

because $h_{j}$ is concave and $\theta G(\theta)$ is strictly increasing. Additionally, it follows from $\theta G(\theta)$ being strictly increasing and $1 / G(\theta)$ being strictly convex that $\theta G(\theta)$ is strictly concave, which implies that

$$
0<G\left(\theta_{j}\left(\boldsymbol{p}^{*}\right)\right)+\theta_{j}\left(\boldsymbol{p}^{*}\right) G^{\prime}\left(\theta_{j}\left(\boldsymbol{p}^{*}\right)\right)<G\left(\theta_{j}\left(\boldsymbol{p}^{* *}\right)\right)+\theta_{j}\left(\boldsymbol{p}^{* *}\right) G^{\prime}\left(\theta_{j}\left(\boldsymbol{p}^{* *}\right)\right)
$$

for any $j$ such that $\theta_{j}\left(\boldsymbol{p}^{*}\right)>\theta_{j}\left(\boldsymbol{p}^{* *}\right)$. The only possibility for both conditions in (21) to be satisfied for every $j$ such that $\theta_{j}\left(\boldsymbol{p}^{*}\right)>\theta_{j}\left(\boldsymbol{p}^{* *}\right)$ is therefore that $\left|\frac{\partial \theta_{j}\left(\boldsymbol{p}^{*}\right)}{\partial p_{j}}\right|>\left|\frac{\partial \theta_{j}\left(\boldsymbol{p}^{* *}\right)}{\partial p_{j}}\right|$ for all such sellers. But, again using convexity of $1 / G(\theta)$ and $p_{j}^{*}<p_{j}^{* *}$ one can show that this is impossible. See the proof in the appendix for the details.

Proposition 6 Suppose that $G$ is continuously differentiable, strictly decreasing and convex and that $1 / G$ is strictly convex, that $\theta G(\theta)$ is strictly increasing in $\theta$, that each $h_{j}$ is increasing, positive valued and concave, and consider the simultaneous move game with payoff functions given by (14). Then, there exists a unique equilibrium.

The reader should keep in mind that in the standard model, uniqueness is within the class of equilibria where buyers use identical symmetric continuation strategies. In contrast, the equilibrium is globally unique in the model with Poisson distributed buyers. One can also note that Proposition 5 does not require $h_{j}(\cdot)$ to be concave, and if one nevertheless can establish a unique symmetric equilibrium (say by a direct computation) then mixed strategies can still be ruled out and the symmetric equilibrium is the only equilibrium in the model. This could be useful in many applications. 10

\footnotetext{
${ }^{10}$ One can also note that this provides a more general existence result than in Galeanios and Kircher [5].
} 


\section{$5 \quad$ Equilibrium with Poisson Buyers and a Fixed Set of Sellers}

In this section I consider the case with $m$ sellers who assign a zero reservation value of the product. The reason for this is twofold. Firstly, it allows me to identify why handling entry is difficult with a known finite set of firms. Secondly, the analysis illustrates how the setup with Poisson distributed buyers is considerably more tractable than the standard finite model. The model also provide some useful intuition for the general characterization in Propositions 5 and 6.I

It is easy to check that $G(\theta)=\frac{1-e^{-\theta n}}{\theta n}$ is strictly decreasing, strictly convex, that $1 / G(\theta)$ is strictly convex and that $\theta G(\theta)=1-e^{-\theta n}$ is strictly increasing in $\theta$. Hence, in the model with Poisson distributed buyers and a given set of sellers labeled $j=\{1, . ., s\}$, it follows from Proposition 3 that the profit function

$$
\pi_{j}(\mathbf{p})=p_{j} n \theta_{j}(\boldsymbol{p}) G\left(\theta_{j}(\boldsymbol{p})\right)=p_{j}\left(1-e^{-\theta_{j}(\boldsymbol{p}) n}\right)
$$

is strictly concave in $p_{j}$ in the range where the seller is visited with positive probability. An equilibrium is defined along the lines of a subgame perfect equilibrium in a standard dynamic game.

Definition 2 The pair $\left(\boldsymbol{\theta}, \boldsymbol{p}^{*}\right)$ with $\boldsymbol{\theta}: R_{+}^{s} \rightarrow \Delta^{s}$ and $\boldsymbol{p}^{*} \in R_{+}^{s}$ is an equilibrium (in buyer symmetric continuation strategies) if:

1. The buyer visitation strategy is optimal for each $\boldsymbol{p} \in R_{+}^{s}$. That is, for each $\mathbf{p}$ the distribution $\theta(\mathbf{p}) \in \Delta^{s}$ is the unique randomization satisfying

$$
\frac{\left(1-p_{i}\right)\left(1-e^{-\theta_{j}(\mathbf{p}) n}\right)}{\theta_{i}(\mathbf{p}) n}=\frac{\left(1-p_{j}\right)\left(1-e^{-\theta_{j}(\mathbf{p}) n}\right)}{\theta_{j}(\mathbf{p}) n}
$$

for each $(i, j)$ such that $\theta_{i}(\mathbf{p})>0$ and $\theta_{j}(\mathbf{p})>0$ and

$$
1-p_{i} \leq \frac{\left(1-p_{j}\right)\left(1-e^{-\theta_{j}(\mathbf{p}) n}\right)}{\theta_{j}(\mathbf{p}) n} .
$$

for each $(i, j)$ such that $\theta_{i}(\mathbf{p})=0$ and $\theta_{j}(\mathbf{p})>0$.

2. Seller optimality: $p_{i}^{*} \in \arg \max _{p_{i}} p_{i}\left(1-e^{-\theta_{i}\left(p_{i}, \mathbf{p}_{-i}^{*}\right) n}\right)$ for each $i \in\{1, \ldots, s\}$.

Recall that, by Proposition 1, the restriction to symmetric continuation strategies is irrelevant for buyer and seller continuation payoffs, so the set of prices supportable using symmetric continuation strategies is the same as if allowing different types coordinate differently.

\subsection{The Unique Equilibrium}

Propositions 5 and 6 apply, so we know that there will a unique equilibrium and that it must be symmetric. This can be understood more intuitively in this more specific model as the profit function in (23) is strictly concave in the range where firm $j$ is visited with positive probability. Given any $\mathbf{p}_{-j}$ there will be some $\bar{p}_{j}\left(\mathbf{p}_{-j}\right)$ such that $j$ is visited with positive probability if 
$p_{j} \in\left[0, \bar{p}_{j}\left(\mathbf{p}_{-j}\right)\right)$, so it follows that the profit function is concave on $\left[0, \bar{p}_{j}\left(\mathbf{p}_{-j}\right)\right]$. However, the profit is flat at zero on $\left[\bar{p}_{j}\left(\mathbf{p}_{-j}\right), 1\right]$, while it was decreasing at $\bar{p}_{j}\left(\mathbf{p}_{-j}\right)$, which creates a nonconcavity. However, it cannot be a best response to price in a way so that no buyer will visit, so if we restrict attention to pure strategy equilibria this non-concavity is irrelevant. The supermodularity guarantees existence of equilibria in pure strategies, and with a unique pure strategy equilibrium there cannot be any mixed strategy equilibrium since the unique equilibrium is the unique rationalizable strategy.

Hence, the first order condition for (23) is necessary and sufficient for optimality given a pure pricing strategy profile $\mathbf{p}_{-j}$ by the other sellers. The first order condition may be expressed as

$$
1-e^{-\theta_{j}(\boldsymbol{p}) n}+p_{j} e^{-\theta_{j}(\boldsymbol{p}) n} \frac{\partial \theta_{j}(\boldsymbol{p})}{\partial p_{j}} n=0
$$

where $\frac{\partial \theta_{j}(\boldsymbol{p})}{\partial p_{j}}$ is given by (16) with $G(\theta)=\left(1-e^{-\theta n}\right) / \theta n$ and

$$
G^{\prime}(\theta)=\frac{1-e^{-\theta n}(1+\theta n)}{(\theta n)^{2}}
$$

Therefore, in a symmetric equilibrium $\mathbf{p}^{*}$ with $p_{j}^{*}=p^{*}$ for each $j$ we have that $\theta_{j}\left(\mathbf{p}^{*}\right)=\frac{1}{s}$ for every $j$ and consequently

$$
\frac{\partial \theta_{j}\left(\mathbf{p}^{*}\right)}{\partial p_{j}}=\frac{G(1 / s)}{\left(1-p^{*}\right) G^{\prime}(1 / s)} \frac{s-1}{s}=\frac{\frac{1-e^{-\theta n}}{\theta n}}{\left(1-p^{*}\right) \frac{1-e^{-\theta n}(1+\theta n)}{(\theta n)^{2}} n} \frac{s-1}{s} .
$$

Substituting $\theta_{j}(\boldsymbol{p})=\frac{1}{s}$ and (26) into (24) and simplifying the result we obtain

$$
p^{*}=\frac{1-e^{-\frac{n}{s}}\left(1+\frac{n}{s}\right)}{1-e^{-\frac{n}{s}}\left[1+\frac{n}{s^{2}}\right]} \rightarrow 1-\frac{\rho}{e^{\rho}-1}
$$

as $n, s \rightarrow \infty$ and $\frac{n}{s} \rightarrow \rho$. Thus, as one would expect, the unique symmetric equilibrium price converges to the price of a natural continuum analogue of the model as the number of buyers and sellers go out of bounds. Hence, the analysis of symmetric equilibria is qualitatively like in the model considered in Burdett et al [2] and the limiting price is identical, which should be rather intuitive because of the Poisson limit theorem. It should, however, be stressed that the Poisson limit relies on the "law of rare events", that is, each buyer visits a seller with a vanishing probability. This rules out equilibria with coordination, which also exist in the binomial model. In contrast, the price in (27) is the unique symmetric equilibrium price, and by appeal to Proposition 5, the unique equilibrium

\subsubsection{The Equilibrium Matching Function}

One important point in Burdett et al [2] is that the matching function is an equilibrium object, whereas in traditional search models it is considered a primitive of the model. Moreover, the model's equilibrium matching function derived using symmetric buyer continuation strategies has 
decreasing returns to scale, which is in stark contrast to the standard search literature that usually assumes constant returns to scale in the matching process. One can note that the closest analogue to an equilibrium matching function in the model with Poisson distributed buyers is $1-e^{-\frac{n}{s}} / \frac{n}{s}$, which satisfies constant returns to scale. The caveat is that this $n$ is the expected number of buyers, so this is the expected matching function, whereas for any realization of the number of buyers the ex post matching function has the same properties as in Burdett et al [2]. ${ }^{11}$

\subsection{What Drives Uniqueness?}

In this section I will use the specifics of the Poisson model to discuss the intuition for why the strategic complementarities can be used exploited to get unique equilibria in these models. In the previous literature, the non-concavity in the profit function created by the kink at $\bar{p}_{i}\left(\mathbf{p}_{-i}\right)$ has been an obstacle. However, the non-concavity is clearly irrelevant in an equilibrium in which all sellers play pure strategies, because attracting no buyers is the worst possible outcome. Indeed, most papers simply restrict attention to equilibria in pure strategies.

Supermoduarity is nice because it immediately establishes existence of pure strategy Nash equilibria, because the least and the largest equilibria are both attained by iterated elimination of strictly dominated strategies. An intuition for this comes from thinking about a symmetric environment. Suppose that we there exists an equilibrium with randomizations, and consider the agent who sets the lowest price with positive probability. This agent will be visited with positive probability irrespective of which prices of the other sellers are realized, so this lowest price must be in the region where there is strict concavity. Hence, it is a global maximizer. Now, consider the other sellers. We know that they all face the same problem as the seller playing the pure strategy except that the roles of the players are switched. But, decreasing the price charged by an opponent decreases the set of maximizers, so these sellers should charge even lower prices.

This symmetry together with the strategic complementarity is also the intuitive reason for uniqueness. Given that equilibria are pure we may write

$$
\beta_{j}\left(\mathbf{p}_{-j}\right)=\arg \max _{p_{j}} \pi_{j}\left(p_{j}, \boldsymbol{p}_{-j}\right),
$$

for the best responses, where we note that $\beta_{j}\left(\mathbf{p}_{-j}\right)$ is unique by the strict concavity and that $\beta_{j}(\cdot)$ is strictly increasing in each $p_{k}$ in the range where seller $k$ is visited with positive probability. This can in this case easily be verified directly as $G(\theta)=\left(1-e^{-\theta n}\right) / \theta n$ satisfies the conditions in Proposition 4, implying that we can differentiate the first order condition associated with (28) with respect to $p_{k}$ to obtain

$$
\frac{\partial \beta_{j}\left(\mathbf{p}_{-j}\right)}{\partial p_{k}}=-\frac{\frac{\partial^{2} \pi_{j}\left(\mathbf{p}_{-j}, \beta_{j}\left(\mathbf{p}_{-j}\right)\right)}{\partial p_{j} \partial p_{k}}}{\frac{\partial^{2} \pi_{j}\left(\mathbf{p}_{-j}, \beta_{j}\left(\mathbf{p}_{-j}\right)\right)}{\partial p_{j}^{2}}}>0,
$$

where the denominator is negative as profit functions are strictly concave in the relevant range. Using symmetry and the fact that prices are strategic complements we can now rule out any

\footnotetext{
${ }^{11}$ I thank Garth Baughman for clarifying this issue.
} 
asymmetric equilibria. The reasoning is simple: If seller $i$ posts a lower price than seller $j$ then seller $i$ faces a distribution of competing prices that is identical to the one for seller $j$ except that $p_{j}$ is replaced with $p_{i}$ in the best response problem for firm $j$. As $p_{i}<p_{j}$ and the best reply is increasing in the price of any competitor, it follows that firm $j$ should optimally post a lower price, which is a contradiction. It has already been shown by direct calculation in Section 5.1 that there is a unique symmetric equilibrium where all sellers price in accordance with (27), so this proves uniqueness of equilibria. More formally, suppose that $\boldsymbol{p}^{*}$ is an equilibrium and that $p_{i}^{*}>p_{j}^{*}$. Let $p_{-i, j}$ denote all prices but $p_{i}$ and $p_{j}$ and write the equilibrium conditions for $i$ and $j$ as

$$
\begin{aligned}
& p_{i}^{*}=\beta_{i}\left(p_{-i, j}^{*}, p_{j}^{*}\right) \\
& p_{j}^{*}=\beta_{j}\left(p_{-i, j}^{*}, p_{i}^{*}\right) .
\end{aligned}
$$

But the best response functions are identical so that $\beta_{i}\left(\boldsymbol{p}_{-i}\right)=\beta_{j}\left(\boldsymbol{p}_{-i}\right)$, so from the fundamental theorem of calculus we obtain

$$
p_{j}^{*}=\beta_{j}\left(p_{-i, j}^{*}, p_{i}^{*}\right)=p_{j}^{*}=\beta_{j}\left(p_{-i, j}^{*}, p_{j}^{*}\right)+\int_{p_{j}^{*}}^{p_{i}^{*}} \frac{\partial \beta_{j}\left(p_{-i, j}^{*}, p_{j}\right)}{\partial p_{j}}=p_{i}^{*}+\int_{p_{j}^{*}}^{p_{i}^{*}} \frac{\partial \beta_{j}\left(p_{-i, j}^{*}, p_{j}\right)}{\partial p_{j}}>p_{i}^{*},
$$

a contradiction.

\section{Entry}

\subsection{Entry with a Known Set of Potential Entrants}

There are at least two ways one can approach entry decisions in directed search. One version would be to interpret the entry cost as some sort of investment decision to "set up shop". In this case, it is perfectly reasonable to assume that all entrants pay the entry cost first and set prices after costs are sunk and the number of entrants is observed by every seller in the market. Modeling entry in this fashion is completely unproblematic. To see this, define the unique equilibrium profit of a seller when $m$ sellers compete as $\Pi(m)$. As $\Pi$ is strictly increasing, equilibrium entry is then determined as the unique $m$ satisfying

$$
\Pi(m+1) \leq K \leq \Pi(m),
$$

where $K$ is the cost of entry.

However, there are circumstances where this "sunk cost" approach may be inappropriate, so that it makes more sense to make entry and pricing decisions simultaneously. In a more general model where there is market segmentation, it may also be more natural to assume that entry and posting the contract are simultaneous. In such a model the equilibrium entry condition is no longer given by (32). The reason is that if $m$ sellers enter in a candidate equilibrium and an additional seller considers deviating, the relevant optimization problem is to solve

$$
\max _{p_{j}} p_{j}\left(1-e^{-\theta_{j}\left(p_{j}, \mathbf{p}^{*}(m)\right) n}\right)
$$


where $\mathbf{p}^{*}(m)$ is the equilibrium price profile with $m$ sellers posting the price in (27). In contrast, if exactly $m+1$ sellers enter in equilibrium, each seller best responds to $m$ other sellers posting the equilibrium price with $m+1$ sellers posting the price in (27). But, this is a lower price than when $m$ sellers enter, so the surprise entrant solving (33) makes a strictly higher profit than if $m+1$ sellers enter in equilibrium.

A similar issue arises in models where different players take on different roles in equilibrium, such as a finite version of Galenianos and Kircher [4]. In the simplest version of this model, firms specialize as high wage or low wage firms respectively. Assuming a continuum of workers and firms the equilibrium condition that determines the proportion of high wage firms is an indifference condition. With a finite set of firms, there is again a situation where a deviant firm that "surprises the market" by switching from one market segment to the other is better off than a firm that plays an equilibrium against the same set of competitors. This creates a problem because if there is an equilibrium with $m$ high wage firms it must be that no high or low wage firm has an incentive to unexpectedly switch to take the other role. But, a deviating firm faces a different optimization problem than firms do when playing in accordance to a candidate equilibrium. As a result, a pure strategy equilibrium cannot be guaranteed to exist.

\subsection{Entry with Randomly Drawn Potential Entrants}

I now return to the full model as specified in Section 2, in which both buyers and sellers are distributed in accordance with independent Poisson distributions. Each seller faces a strictly positive cost of entry $K$, which is sufficiently large to guarantee a non-trivial entry decision. We note that a seller strategy is a map $(a, p): T \rightarrow[0,1] \times R_{+}$where $\alpha_{t}$ is the probability that a seller of type $t$ enters and $p_{t}$ is the price posted by type $t$ conditional on entry. Sellers could of course in principle also randomize over prices, but as this is not possibly part of any equilibrium I have immediately imposed a pure pricing strategy.

We note that the decomposition and aggregation property imply that the total number of firms that enter is a Poisson with expected queue length $\sum_{t} \alpha_{t} m_{t}$. This follows from the same calculations as in (2) and (1). We also note that the profit function in (10) is the same for every type of seller, which is yet another consequence of the environmental equivalence implied by the Poisson distribution. Finally, we note that Proposition 3 implies that the profit function in (10) is strictly concave in its own price in the range where the seller is visited for sure. However, for asymmetric pricing strategies, the non-concavity implied by the kink created when no buyers arrive may be relevant with positive probability, so me must use a somewhat different argument than for the case with a known set of sellers to rule out asymmetric equilibria.

Proposition 7 In any equilibrium there exists a unique equilibrium price $p^{*}$ such that $p_{t}^{*}=p^{*}$. Moreover, if $\left(a_{1}^{*}, \ldots, a_{T}^{*}\right)$ is an equilibrium entry profile there exists $\alpha^{*}$ such that $\alpha_{t}^{*}=\alpha^{*}$ for each $t$ such that all buyers and sellers earn the same payoffs as in the equilibrium with asymmetric entry. Proof. Let $\mathbf{p}^{*}=\left(p_{1}^{*}, \ldots, p_{T}^{*}\right)$ and $\boldsymbol{\alpha}^{*}=\left(\alpha_{1}^{*}, \ldots, \alpha_{T}^{*}\right)$ be an equilibrium price and entry strategy, which is true if and only if $p_{t}^{*}$ maximizes (10) for each $t$ and each type seller is indifferent between 
entry and staying out. From Proposition 3 we know that each $p_{t}\left(1-e^{-\theta_{t}\left(p_{t}, \mathbf{p}^{*}, k\right) n}\right)$ is strictly concave in $p$ in some range $\left[0, \bar{p}\left(\mathbf{p}^{*}\right)\right]$, but has a non-concave range where no buyers visit the firm. However, all active sellers face the same optimization problem with the same set of maximizers. Let $p_{\text {min }}^{*}$ be the smallest maximizer. Obviously, a seller posting $p_{\min }^{*}$ must be visited with a strictly positive probability regardless of which sellers enter, so $p_{\min }^{*}$ is in the range where the all profit functions in the support of the expected profit function are strictly concave, implying that (10) is strictly concave in a range around $p_{\min }^{*}$. We also have that the expected profit function is single peaked, so $p_{\min }^{*}$ must be the unique global maximum. But all sellers face the same optimization problem, so there must be a unique price $p^{*}$ such that $p_{t}^{*}=p^{*}$ for each $t$ in any equilibrium. Since all types post the same price only the total number of entrants matter. This is distributed Poisson with parameter $\sum_{t \in T} \alpha_{t}^{*} m_{t}$ implying that there is a symmetric randomization probability

$$
\alpha^{*}=\frac{\sum_{t \in T} \alpha_{t}^{*} m_{t}}{\sum_{t \in T} m_{t}}
$$

that is consistent with equilibrium.

Proposition (7) establishes that any equilibrium is payoff equivalent to a symmetric equilibrium. It is worth noting that this argument is considerably simpler than establishing symmetry with a known set of sellers. ${ }^{1213}$

Imposing symmetry, write $p$ for a generic price, $p^{*}$ for a symmetric equilibrium price and $\theta_{k}\left(p, p^{*}\right)$ for the probability that a buyer visits a seller posting price $p$ given that $k$ other sellers post $p^{*}$. The pricing problem for a seller that enters can then be expressed as

$$
\max _{p} \sum_{k=0}^{\infty} \frac{e^{-(\alpha m)}(\alpha m)^{k}}{k !} p_{i}\left(1-e^{-\theta_{k}\left(p, p^{*}\right) n}\right) .
$$

Proposition (7) implies that any equilibrium price vector is symmetric and that the profit function is locally strictly concave around symmetry, so the first order condition

$$
\sum_{k=0}^{\infty} \frac{e^{-(\alpha m)}(\alpha m)^{k}}{k !}\left(1-e^{-\theta_{k}\left(p, p^{*}\right) n}+p^{*} e^{-\theta_{k}\left(p, p^{*}\right) n} \frac{\partial \theta_{k}\left(p^{*}, p^{*}\right)}{\partial p} n\right)=0
$$

is necessary and sufficient. Using symmetry, we have that $\theta_{k}\left(p, p^{*}\right) n=\frac{n}{k+1}$ for each $k \geq 0$ and that

$$
\frac{\partial \theta_{k}\left(p^{*}, p^{*}\right)}{\partial p} n=-\frac{\frac{n}{k+1}\left(1-e^{-\frac{n}{k+1}}\right)}{\left(1-p^{*}\right)\left(1-e^{-\frac{n}{k+1}}\left(1+\frac{n}{k+1}\right)\right)} \frac{k}{k+1},
$$

\footnotetext{
${ }^{12}$ For ease of exposition the proof of Proposition 7 uses the demand system defined from Poisson distributed buyers. The result holds also if the profit is on the general form $p_{t} \theta_{i}\left(p_{t}, \mathbf{p}^{*}, k\right) G\left(\theta_{i}\left(p_{t}, \mathbf{p}^{*}, k\right)\right)$ under the conditions in Proposition 3. The proof is the same as the only property if the demand system that is used is that the profit function is strictly concave when the seller is visited with prositive probability.

${ }^{13}$ Jehiel and Lamy [9] uses a Poisson entry game for similar reasons.
} 
so we can solve for the candidate equilibrium price in closed form,

$$
p^{*}(\alpha)=\frac{\sum_{k=0}^{\infty} \frac{e^{-(\alpha m)}(\alpha m)^{k}}{k !}\left(1-e^{-\frac{n}{k+1}}\right)}{\sum_{k=0}^{\infty} \frac{e^{-(\alpha m)}(\alpha m)^{k}}{k !} \frac{\left(1-e^{-\frac{n}{k+1}}\right)}{\left(1-e^{-\frac{n}{k+1}}\left(1+\frac{n}{k+1}\right)\right)}\left[1-e^{-\frac{n}{k+1}}\left(1+\frac{n}{(k+1)^{2}}\right)\right]} .
$$

Given any $\alpha \in[0,1]$ we have that $p^{*}(\alpha)$ is uniquely defined, so we can immediately conclude that there is a unique candidate equilibrium price for every entry probability $\alpha$. However, in order to show that there is a unique equilibrium price given any cost of selling $K$ we need to establish that $p^{*}(\alpha)$ is monotone decreasing in $\alpha$ as otherwise sellers could be indifferent between participating and staying out both at a low price and low entry equilibrium and also at a high price high entry equilibrium.

Let $H: G_{+} \rightarrow R_{+}$and $H: R_{+} \rightarrow R_{+}$be defined as

$$
\begin{aligned}
G(k) & =1-e^{-\frac{n}{k+1}} \\
H(k) & =\frac{\left(1-e^{-\frac{n}{k+1}}\right)}{\left(1-e^{-\frac{n}{k+1}}\left(1+\frac{n}{k+1}\right)\right)}\left[1-e^{-\frac{n}{k+1}}\left(1+\frac{n}{(k+1)^{2}}\right)\right]
\end{aligned}
$$

To demonstrate that $p^{*}(\alpha)$ is monotone in $\alpha$ we show that.

Lemma $2 G(\cdot)$ is strictly decreasing and $H(\cdot)$ is strictly increasing on $R_{+}$.

The proof, a somewhat complicated direct computation, is relegated to the appendix. Using the monotonicity of $G$ and $H$ we are in a position where we can demonstrate that the price is strictly decreasing in the entry probability, as one would expect.

Lemma $3 p^{*}(\cdot)$ defined in (38) is strictly decreasing in $\alpha$.

Proof. Suppose that $a^{\prime}<a^{\prime \prime}$. Then the Poisson with probability mass density $\frac{e^{-\left(\alpha^{\prime} m\right)}\left(\alpha^{\prime} m\right)^{k}}{k !}$ is first order stochastically dominated by the Poisson with probability mass density $\frac{e^{-\left(\alpha^{\prime \prime} m\right)}\left(\alpha^{\prime \prime} m\right)^{k}}{k !}$. Since $G(k)$ is strictly decreasing, stochastic dominance implies

$$
\sum_{k=1}^{\infty} \frac{e^{-\left(\alpha^{\prime} m\right)}(\alpha m)^{k}}{k !}\left(1-e^{-\frac{n}{k+1}}\right)>\sum_{k=1}^{\infty} \frac{e^{-\left(\alpha^{\prime \prime} m\right)}(\alpha m)^{k}}{k !}\left(1-e^{-\frac{n}{k+1}}\right)
$$

and since $H$ is strictly decreasing dominance implies

$$
\begin{aligned}
& \sum_{k=1}^{\infty} \frac{e^{-\left(\alpha^{\prime} m\right)}(\alpha m)^{k}}{k !} \frac{\left(1-e^{-\frac{n}{k+1}}\right)}{\left(1-e^{-\frac{n}{k+1}}\left(1+\frac{n}{k+1}\right)\right)}\left[1-e^{-\frac{n}{k+1}}\left(1+\frac{n}{(k+1)^{2}}\right)\right] \\
\leq & \sum_{k=1}^{\infty} \frac{e^{-\left(\alpha^{\prime \prime} m\right)}(\alpha m)^{k}}{k !} \frac{\left(1-e^{-\frac{n}{k+1}}\right)}{\left(1-e^{-\frac{n}{k+1}}\left(1+\frac{n}{k+1}\right)\right)}\left[1-e^{-\frac{n}{k+1}}\left(1+\frac{n}{(k+1)^{2}}\right)\right]
\end{aligned}
$$

which implies that $p^{*}\left(a^{\prime}\right)>p^{*}\left(a^{\prime \prime}\right)$.

Since the price is monotonic in the entry probability, it follows immediately that there is a unique symmetric solution to the entry problem, so we have established that 
Corollary 1 For any $K>0$ there is a unique entry probability $\alpha(K)$ that is consistent with an equilibrium in symmetric entry strategies. Moreover if

$$
\frac{\left[e^{-m}+\sum_{k=1}^{\infty} \frac{e^{-(m)}(m)^{k}}{k !}\left(1-e^{-\frac{n}{k+1}}\right)\right]^{2}}{e^{-m}+\sum_{k=1}^{\infty} \frac{e^{-(m)}(m)^{k}}{k !} \frac{\left(1-e^{-\frac{n}{k+1}}\right)}{\left(1-e^{-\frac{n}{k+1}}\left(1+\frac{n}{k+1}\right)\right)}\left[1-e^{-\frac{n}{k+1}}\left(1+\frac{n}{(k+1)^{2}}\right)\right]}<K<1,
$$

then $\alpha(K) \in(0,1)$ and every seller that enters is indifferent between entering and staying out.

If sellers have payoff-irrelevant characteristics to condition decisions on one can construct equilibria with some types entering with a higher probability than others, but the average probability of entry is uniquely defined, so the equilibrium is unique in terms of both buyer and seller payoffs.

It is interesting to note that when inequality (42) is satisfied the distribution of active sellers is unchanged as $m$ increases. That is, the resulting decrease in the entry probability exactly cancels the effect from increasing the expected number of sellers. This is yet another illustration of how the Poisson distribution differs qualitatively from having a fixed set of players. In Levin and Smith [14], a similar type of equilibrium is constructed in a model of auctions with entry, but they find that increasing the set of potential bidders can decrease the efficiency of the market even if the expected number of bidders is non-decreasing. This result stems from coordination costs being increasing in the number of potential bidders, essentially for the same reason as there are decreasing returns to scale in the matching function when there is a given number of buyers and sellers. In contrast, modelling the number of potential sellers as a Poisson distribution creates constant returns, which implies that the coordination costs are neutral to the number of potential sellers in the range where the entry probability is below unity.

\subsubsection{Convergence Towards the Market Utility Benchmark}

With some further notational abuse I write $\alpha(K, n, m)$ for the unique (average) randomization probability when the cost of selling is $K$ and there are $n$ buyers and $m$ potential sellers. While $p^{*}(\alpha(K, n, m))$ in (38) is a deterministic number that can be thought of as an expectation (and not a random variable), it turns out that it is useful to note that $G(k)$ and $H(k)$ converge in probability in order to derive the (deterministic) limit of $p^{*}(\alpha(K, n, m))$ as $n, m \rightarrow \infty$ and $\frac{n}{m} \rightarrow \rho$.

That is, we use the fact that the number of active sellers, $\frac{k}{m}$, converges in probability to $\alpha(K, n, m)$ in order to derive the limiting price and expected equilibrium profit. More formally, Chebyshev's inequality implies that

$$
\operatorname{Pr}[|k-\alpha(K, n, m) m|>\delta m] \leq \frac{\sigma^{2}}{\delta^{2} m^{2}}=\frac{\alpha(K, n, m)}{\delta^{2} m}
$$

holds for any $m$, because $\sigma^{2}=\alpha(K, n, m) m$ is the variance of the number of active sellers $k$ as $\alpha(K, n, m) m$ is both the first and the second moment of the Poisson generated when the expected 
number of potential sellers is $m$ and every seller enters with probability $\alpha(K, n, m)$.It follows that if $\frac{n}{m}=\rho$ we have that

$$
\operatorname{Pr}\left[\left|\frac{k}{n}-\frac{\alpha(K, n, m)}{\rho}\right|>\frac{\delta}{\rho}\right] \leq \frac{\alpha(K, n, m)}{\delta^{2} m} \rightarrow 0
$$

as $m \rightarrow \infty$. More generally, if $n, m \rightarrow \infty, \frac{n}{m} \rightarrow \rho$, and $\alpha(K, n, m) \rightarrow \alpha^{*}$ (taking a subsequence if necessary) we have that $\frac{k}{n}$ converges in probability to $\frac{\alpha^{*}}{\rho}$. Hence,

$$
p^{*}(\alpha(K, n, m)) \rightarrow \frac{\left(1-e^{-\frac{\rho}{\alpha^{*}}}\right)}{\left(1-e^{-\frac{\rho}{\alpha^{*}}}\right)\left[\frac{1-e^{-\frac{\rho}{\alpha^{*}}}}{\left(1-e^{-\frac{\rho}{\alpha^{*}}}\left(1+\frac{\rho}{\alpha^{*}}\right)\right)}\right]}=\frac{1-e^{-\frac{\rho}{\alpha^{*}}}\left(1+\frac{\rho}{\alpha^{*}}\right)}{1-e^{-\frac{\rho}{\alpha^{*}}}}=1-\frac{\frac{\rho}{\alpha^{*}}}{e^{\frac{\rho}{\alpha^{*}}}-1},
$$

as $n, m \rightarrow \infty, \frac{n}{m} \rightarrow \rho$, and $\alpha(K, n, m) \rightarrow \alpha^{*}$. Also note that as $\frac{k}{n}$ converges in probability to $\frac{\alpha^{*}}{\rho}$, we have that $e^{-\frac{k}{n}}$ converges in probability to $e^{-\frac{\rho}{\alpha^{*}}}$, implying that the equilibrium expected profit

$$
\pi\left(p^{*}(\alpha(K, n, m)), p^{*}(\alpha(K, n, m)), \alpha(K, n, m)\right) \rightarrow 1-e^{-\frac{\rho}{\alpha^{*}}}\left(1+\frac{\rho}{\alpha^{*}}\right)=K
$$

along any convergent subsequence. Since $1-e^{-x}(1+x)$ is strictly increasing, there is a unique solution $\alpha^{*}$ to $1-e^{-\frac{\rho}{\alpha^{*}}}\left(1+\frac{\rho}{\alpha^{*}}\right)=K$ implying that $\{\alpha(K, n, m)\}$ is a convergent sequence. Hence, the right hand side of (46) is the limiting equilibrium profit. Also notice that the right hand side of (46) is identical to the profit in the market utility model and that the limiting price in (45) corresponds to the equilibrium price (27) for the equilibrium active buyer to seller ratio. We conclude that the market utility benchmark can indeed be seen as the limit a large finite model in which both buyers and sellers are distributed in accordance with a Poisson.

\section{Concluding Remarks}

The results of this paper suggest that drawing players randomly from a Poisson distribution can be a useful compromise between a market utility model and a standard finite directed search model. In the simplest possible setup, I demonstrated that there is no longer any need to make an equilibrium selection and that an otherwise intractable entry problem can be handled without too much difficulty. The added tractability should also be helpful in richer models where buyers or sellers have payoff relevant types, and or in models where contracts are more elaborate than posting a single price, which is a topic for future research.

I have also connected the directed search literature with the literature on supermodular games. Using a set of conditions previously used by Galenianos and Kircher [5] and Kim and Camera [11] I showed that prices are strategic complements in the sense of Milgrom and Shannon [15]. This more or less immediately implies that the equilibrium is unique as long as there is a unique symmetric pure strategy equilibrium. More generally, it also implies that there is a smallest and a largest equilibrium, both in pure strategies. This makes it relatively easy to rule out mixing in any equilibrium, which has been an issue in the previous literature. With heterogeneous profit functions 
for the sellers some work is needed, but working directly with the necessary condition for optimality I construct a rather simple proof of uniqueness of equilibria.

Supermodularity should also be useful as it generates monotone comparative statics, which should be a useful observation for the applied literature.

\section{References}

[1] Bland, J. and S. Loertscher (2012), "Monotonicity, Non-Participation, and Directed Search Equilibria," mimeo, University of Melbourne.

[2] Burdett, K. Shi S., and R. Wright, (2001). "Pricing and Matching with Frictions," Journal of Political Economy, 109(5), October 2001, 1060-1085.

[3] Choi, M. (2015). Monotone Comparative Statics for Directed Search Models. mimeo, University of Iowa..

[4] Galenianos, M. and P. Kircher, (2009) "Directed Search with Multiple Job Applications," Journal of Economic Theory, 144(2), 2009, 445-471.

[5] Galenianos, M. and P. Kircher, (2012) "On the Game-theoretic Foundations of Competitive Search Equilibrium," International Economic Review, 53(1), 1-21.

[6] Geromichalos, A. (2012) "Directed search and optimal production," Journal of Economic Theory, 47(6), Pages 2303-2331.

[7] Gonzalez, Francisco M., and Shouyong Shi. (2010) "An equilibrium theory of learning, search, and wages." Econometrica 78.2: 509-537.

[8] Haight, F.A., (1967), Handbook of the Poisson Distribution, New York, Wiley.

[9] Jehiel, Philippe, and Laurent Lamy.(2014) "On discrimination in procurement auctions." mimeo, Paris School of Economics.

[10] Julien, B., J. Kennes and I. King, (2000), "Bidding for labor," Review of Economic Dynamics, 3(4), 619-649.

[11] Kim, J. and G. Camera (2014). "Uniqueness of equilibrium in directed search models," Journal of Economic Theory 151, 248-267.

[12] Lester, B., (2011) "Information and Prices with Capacity Constraints," American Economic Review, 101(4), 1591-1600.

[13] Lester, B, Visschersz L., and R. Wolthoff. (2014) "Meeting Technologies and Optimal Trading Mechanisms in Competitive Search Markets," mimeo. 
[14] Levin, Dan, and James L. Smith. "Equilibrium in auctions with entry." The American Economic Review (1994): 585-599.

[15] Milgrom, P., \& Shannon, C. (1994). Monotone comparative statics. Econometrica, 157-180.

[16] Moen, Espen R., (1997) "Competitive search equilibrium." Journal of Political Economy 105.2, 385-411.

[17] Montgomery, J. D. (1991). Equilibrium wage dispersion and interindustry wage differentials. The Quarterly Journal of Economics, 163-179.

[18] Moscarini, G., \& Postel-Vinay, F. (2013). Stochastic search equilibrium. The Review of Economic Studies, rdt012.

[19] Myerson, Roger (1998). "Population Uncertainty and Poisson games". International Journal of Game Theory (27): 375-392.

[20] Myerson, Roger B. (2000). "Large Poisson Games," Journal of Economic Theory 94 (1): 7-45.

[21] Peters, M. (1984). "Bertrand Equilibrium with Capacity Constraints and Restricted Mobility.", Econometrica, 52(5): 1117-27.

[22] Peters, M. (2000), "Limits of Exact Equilibria for Capacity Constrained Sellers with Costly Search," Journal of Economic Theory, 95(2), 139-168.

[23] Shannon, C. (1995). Weak and strong monotone comparative statics. Economic Theory, 5(2), 209-227.

[24] Topkis, D. M. (1978). Minimizing a submodular function on a lattice. Operations research, $26(2), 305-321$.

\section{A Appendix}

\section{A.1 Proof of Proposition 2}

Existence follows using a standard application of Kakutani's theorem along the same lines as Nash existence theorem for finite games. Details are left to the reader.

\section{A.1.1 Proof of Uniqueness}

Proof. Suppose that for some $\boldsymbol{p}$ there are multiple solutions to (12) and (13). Denote these by $\left(\theta_{1}^{\prime}, \ldots, \theta_{s}^{\prime}\right)$ and $\left(\theta_{1}^{\prime \prime}, \ldots, \theta_{s}^{\prime \prime}\right)$. Then there exists at least one $i$ such that $\theta_{i}^{\prime}>\theta_{i}^{\prime \prime}$ and one $j$ such that $\theta_{j}^{\prime}<\theta_{j}^{\prime \prime}$ as randomizations have to add up to unity. Consider the case with $\theta_{i}^{\prime \prime}>0$ and $\theta_{j}^{\prime}>0$. Then since $\theta_{i}^{\prime}>\theta_{i}^{\prime \prime}>0$ and $\theta_{j}^{\prime \prime}>\theta_{j}^{\prime}>0$ there must be indifference between $i$ and $j$ in equilibrium $\left(\theta_{1}^{\prime}, \ldots, \theta_{s}^{\prime}\right)$ as well as in equilibrium $\left(\theta_{1}^{\prime \prime}, \ldots, \theta_{s}^{\prime \prime}\right)$ so that

$$
\left(1-p_{j}\right) G\left(\theta_{j}^{\prime}\right)=\left(1-p_{i}\right) G\left(\theta_{i}^{\prime}\right)<\left(1-p_{i}\right) G\left(\theta_{i}^{\prime \prime}\right)=\left(1-p_{j}\right) G\left(\theta_{j}^{\prime \prime}\right),
$$


where the inequality follows because $G$ is strictly decreasing and $\theta_{i}^{\prime}>\theta_{i}^{\prime \prime}$. But, this is impossible since $\theta_{j}^{\prime \prime}>\theta_{j}^{\prime}$ implies that $G\left(\theta_{j}^{\prime}\right)>G\left(\theta_{j}^{\prime \prime}\right)$, again as $G$ is strictly decreasing. If instead $\theta_{i}^{\prime \prime}>0$ and $\theta_{j}^{\prime}=0$. Then, there is indifference between $i$ and $j$ in equilibrium $\left(\theta_{1}^{\prime \prime}, \ldots, \theta_{s}^{\prime \prime}\right)$, but it may be better to visit $i$ than $j$ in the $\left(\theta_{1}^{\prime}, \ldots, \theta_{s}^{\prime}\right)$ equilibrium. Hence,

$$
\left(1-p_{j}\right) G(0) \leq\left(1-p_{i}\right) G\left(\theta_{i}^{\prime}\right)<\left(1-p_{i}\right) G\left(\theta_{i}^{\prime \prime}\right)=\left(1-p_{j}\right) G\left(\theta_{j}^{\prime \prime}\right)<\left(1-p_{j}\right) G(0),
$$

where the first inequality is equilibrium condition (13), the second is from $G$ being strictly decreasing, the equality is equilibrium condition (12), and the finial inequality is because $G$ is strictly decreasing. The final possibility is $\theta_{i}^{\prime \prime}=0$ and $\theta_{j}^{\prime}=0$, in which case

$$
\begin{aligned}
\left(1-p_{i}\right) G\left(\theta_{i}^{\prime}\right) & \geq\left(1-p_{j}\right) G(0)>\left(1-p_{j}\right) G\left(\theta_{j}^{\prime \prime}\right) \\
\left(1-p_{j}\right) G\left(\theta_{j}^{\prime \prime}\right) & \geq\left(1-p_{i}\right) G(0)>\left(1-p_{i}\right) G\left(\theta_{i}^{\prime}\right)
\end{aligned}
$$

again a contradiction.

\section{A.1.2 Proof of Monotonicity in Own Price}

Since weak monotonicity follows by combining the strict monotonicity in the interior range with the corner solutions I only provide proof for the strict monotonicity results.

Proof. Suppose that the visitation probability is not strictly decreasing in the own price in the range where it is interior, so that $0<\theta_{i}\left(p_{i}^{\prime}, \mathbf{p}_{-i}\right) \leq \theta_{i}\left(p_{i}^{\prime \prime}, \mathbf{p}_{-i}\right)<1$ and $p_{i}^{\prime}<p_{i}^{\prime \prime}$. Then a buyer is strictly better off when the price is $p_{i}^{\prime}$ when going to firm $i$,

$$
\left(1-p_{i}^{\prime}\right) G\left(\theta_{i}\left(p_{i}^{\prime}, \mathbf{p}_{-i}\right)\right)>\left(1-p_{i}^{\prime \prime}\right) G\left(\theta_{i}\left(p_{i}^{\prime \prime}, \mathbf{p}_{-i}\right)\right)
$$

Since the randomizations have to add up there must be some $j$ such that $\theta_{j}\left(p_{i}^{\prime}, \mathbf{p}_{-i}\right) \geq \theta_{j}\left(p_{i}^{\prime \prime}, \mathbf{p}_{-i}\right)$. We note that, since $0<\theta_{i}\left(p_{i}^{\prime}, \mathbf{p}_{-i}\right)<1$, it is without loss of generality to assume that $\theta_{j}\left(p_{i}^{\prime}, \mathbf{p}_{-i}\right)>$ 0 . This follows because if $\theta_{j}\left(p_{i}^{\prime}, \mathbf{p}_{-i}\right)=0$, then $\theta_{j}\left(p_{i}^{\prime \prime}, \mathbf{p}_{-i}\right)=0$, so there must exist some $k \neq$ $i, j$ such that $\theta_{k}\left(p_{i}^{\prime}, \mathbf{p}_{-i}\right) \geq \theta_{k}\left(p_{i}^{\prime \prime}, \mathbf{p}_{-i}\right)$. By induction, the only possibility for no $j$ such that $\theta_{j}\left(p_{i}^{\prime}, \mathbf{p}_{-i}\right) \geq \theta_{j}\left(p_{i}^{\prime \prime}, \mathbf{p}_{-i}\right)$ and $\theta_{j}\left(p_{i}^{\prime}, \mathbf{p}_{-i}\right)>0$ is if $\theta_{j}\left(p_{i}^{\prime}, \mathbf{p}_{-i}\right)=\theta_{j}\left(p_{i}^{\prime \prime}, \mathbf{p}_{-i}\right)=0$ for every $j \neq i$, which contradicts the hypothesis $\theta_{i}\left(p_{i}^{\prime}, \mathbf{p}_{-i}\right)<1$. Hence, condition (12) must hold between $i$ and $j$ given $p_{i}^{\prime}$ so that, using (A4), (12), and (13) we obtain

$$
\begin{aligned}
\left(1-p_{j}\right) G\left(\theta_{j}\left(p_{i}^{\prime}, \mathbf{p}_{-i}\right)\right) & =\left(1-p_{i}^{\prime}\right) G\left(\theta_{i}\left(p_{i}^{\prime}, \mathbf{p}_{-i}\right)\right)>\left(1-p_{i}^{\prime \prime}\right) G\left(\theta_{i}\left(p_{i}^{\prime \prime}, \mathbf{p}_{-i}\right)\right) \\
& \geq\left(1-p_{j}\right) G\left(\theta_{j}\left(p_{i}^{\prime \prime}, \mathbf{p}_{-i}\right)\right)
\end{aligned}
$$

a contradiction as $\theta_{j}\left(p_{i}^{\prime}, \mathbf{p}_{-i}\right) \geq \theta_{j}\left(p_{i}^{\prime \prime}, \mathbf{p}_{-i}\right)$ implies that $G\left(\theta_{j}\left(p_{i}^{\prime}, \mathbf{p}_{-i}\right)\right) \leq G\left(\theta_{j}\left(p_{i}^{\prime \prime}, \mathbf{p}_{-i}\right)\right)$.

\section{A.1.3 Proof of Monotonicity in Competitor's Price}

Again, only the strict part is non-trivial.

Proof. Suppose not so that $\theta_{i}\left(p_{j}^{\prime}, \mathbf{p}_{-j}\right) \geq \theta_{i}\left(p_{j}^{\prime \prime}, \mathbf{p}_{-j}\right)$ and $p_{j}^{\prime}<p_{j}^{\prime \prime}$. Then

$$
0<\left(1-p_{i}\right) G\left(\theta_{i}\left(p_{j}^{\prime}, \mathbf{p}_{-j}\right)\right) \leq\left(1-p_{i}\right) G\left(\theta_{i}\left(p_{j}^{\prime \prime}, \mathbf{p}_{-j}\right)\right)
$$


It follows that $\theta_{k}\left(p_{j}^{\prime}, \mathbf{p}_{-j}\right) \geq \theta_{k}\left(p_{j}^{\prime \prime}, \mathbf{p}_{-j}\right)$ for every $k \neq i, j$ because if $\theta_{k}\left(p_{j}^{\prime}, \mathbf{p}_{-j}\right)<\theta_{k}\left(p_{j}^{\prime \prime}, \mathbf{p}_{-j}\right)$ for some $k$ then

$$
\begin{aligned}
\left(1-p_{i}\right) G\left(\theta_{i}\left(p_{j}^{\prime}, \mathbf{p}_{-j}\right)\right) & \geq\left(1-p_{k}\right) G\left(\theta_{k}\left(p_{j}^{\prime}, \mathbf{p}_{-j}\right)\right)>\left(1-p_{k}\right) G\left(\theta_{k}\left(p_{j}^{\prime \prime}, \mathbf{p}_{-j}\right)\right) \\
& =\left(1-p_{i}\right) G\left(\theta_{i}\left(p_{j}^{\prime \prime}, \mathbf{p}_{-j}\right)\right) .
\end{aligned}
$$

Hence, for the randomizations to add up $\theta_{j}\left(p_{j}^{\prime}, \mathbf{p}_{-j}\right) \leq \theta_{j}\left(p_{j}^{\prime \prime}, \mathbf{p}_{-j}\right)$, which contradicting strict monotonicity of $\theta_{j}$ in $p_{j}$.

\section{A.2 Proof of Lemma 1}

Proof. Suppose $\theta_{j}(\mathbf{p})$ is not strictly concave in $p_{j}$. Then, there exists some exists $\mathbf{p}_{-j}$ and $p_{j}^{\prime}, p_{j}^{\prime \prime}$ and $\lambda$ such that

$$
\theta_{j}\left(\mathbf{p}_{-j}, \lambda p_{j}^{\prime}+(1-\lambda) p_{j}^{\prime \prime}\right) \leq \lambda \theta_{j}\left(\mathbf{p}_{-j}, p_{j}^{\prime}\right)+(1-\lambda) \theta_{j}\left(\mathbf{p}_{-j}, p_{j}^{\prime \prime}\right)
$$

implying that there exists $i \neq j$ such that

$$
\theta_{i}\left(\mathbf{p}_{-j}, \lambda p_{j}^{\prime}+(1-\lambda) p_{j}^{\prime \prime}\right) \geq \lambda \theta_{i}\left(\mathbf{p}_{-j}, p_{j}^{\prime}\right)+(1-\lambda) \theta_{i}\left(\mathbf{p}_{-j}, p_{j}^{\prime \prime}\right)
$$

as otherwise the probabilities would not sum to unity. As $\theta_{i}\left(\mathbf{p}_{-j}, \lambda p_{j}^{\prime}+(1-\lambda) p_{j}^{\prime \prime}\right)>0$ in the range considered, we have that

$$
\begin{aligned}
& \left(1-\lambda p_{j}^{\prime}-(1-\lambda) p_{j}^{\prime \prime}\right) G\left(\theta_{j}\left(\mathbf{p}_{-j}, \lambda p_{j}^{\prime}+(1-\lambda) p_{j}^{\prime \prime}\right)\right) \\
= & \left(1-p_{i}\right) G\left(\theta_{i}\left(\mathbf{p}_{-j}, \lambda p_{j}^{\prime}+(1-\lambda) p_{j}^{\prime \prime}\right)\right) \\
/ G \text { decreasing and }(\mathrm{A} 9) / \leq & \left(1-p_{i}\right) G\left(\lambda \theta_{i}\left(\mathbf{p}_{-j}, p_{j}^{\prime}\right)+(1-\lambda) \theta_{i}\left(\mathbf{p}_{-j}, p_{j}^{\prime \prime}\right)\right) \\
/ G \text { convex } / \leq & \lambda\left(1-p_{i}\right) G\left(\theta_{i}\left(\mathbf{p}_{-j}, p_{j}^{\prime}\right)\right)+(1-\lambda)\left(1-p_{i}\right) G\left(\theta_{i}\left(\mathbf{p}_{-j}, p_{j}^{\prime \prime}\right)\right) \\
/(12) \text { and }(13) / \leq & \lambda\left(1-p_{j}^{\prime}\right) G\left(\theta_{j}\left(\mathbf{p}_{-j}, p_{j}^{\prime}\right)\right)+(1-\lambda)\left(1-p_{i}^{\prime \prime}\right) G\left(\theta_{j}\left(\mathbf{p}_{-j}, p_{j}^{\prime \prime}\right)\right) .
\end{aligned}
$$

Since $\frac{1}{G}$ is increasing and strictly convex it follows that

$$
\begin{aligned}
\frac{1}{G\left(\theta_{j}\left(\mathbf{p}_{-j}, \lambda p_{j}^{\prime}+(1-\lambda) p_{j}^{\prime \prime}\right)\right)} & \leq \frac{1}{G\left(\lambda \theta_{j}\left(\mathbf{p}_{-j}, p_{j}^{\prime}\right)+(1-\lambda) \theta_{j}\left(\mathbf{p}_{-j}, p_{j}^{\prime \prime}\right)\right)} \\
& <\frac{\lambda}{G\left(\theta_{j}\left(\mathbf{p}_{-j}, p_{j}^{\prime}\right)\right)}+\frac{(1-\lambda)}{G\left(\theta_{j}\left(\mathbf{p}_{-j}, p_{j}^{\prime \prime}\right)\right)} \\
& =\frac{\lambda G\left(\theta_{j}\left(\mathbf{p}_{-j}, p_{j}^{\prime \prime}\right)\right)+(1-\lambda) G\left(\theta_{j}\left(\mathbf{p}_{-j}, p_{j}^{\prime}\right)\right)}{G\left(\theta_{j}\left(\mathbf{p}_{-j}, p_{j}^{\prime}\right)\right) G\left(\theta_{j}\left(\mathbf{p}_{-j}, p_{j}^{\prime \prime}\right)\right)}
\end{aligned}
$$

or rearranging

$$
G\left(\theta_{j}\left(\mathbf{p}_{-j}, \lambda p_{j}^{\prime}+(1-\lambda) p_{j}^{\prime \prime}\right)\right)>\frac{G\left(\theta_{j}\left(\mathbf{p}_{-j}, p_{j}^{\prime}\right)\right) G\left(\theta_{j}\left(\mathbf{p}_{-j}, p_{j}^{\prime \prime}\right)\right)}{\lambda G\left(\theta_{j}\left(\mathbf{p}_{-j}, p_{j}^{\prime \prime}\right)\right)+(1-\lambda) G\left(\theta_{j}\left(\mathbf{p}_{-j}, p_{j}^{\prime}\right)\right)}
$$


which combined with (A10) gives

$$
\begin{gathered}
\frac{\left(1-\lambda p_{j}^{\prime}-(1-\lambda) p_{j}^{\prime \prime}\right) G\left(\theta_{j}\left(\mathbf{p}_{-j}, p_{j}^{\prime}\right)\right) G\left(\theta_{j}\left(\mathbf{p}_{-j}, p_{j}^{\prime \prime}\right)\right)}{\lambda G\left(\theta_{j}\left(\mathbf{p}_{-j}, p_{j}^{\prime \prime}\right)\right)+(1-\lambda) G\left(\theta_{j}\left(\mathbf{p}_{-j}, p_{j}^{\prime}\right)\right)} \\
<\lambda\left(1-p_{j}^{\prime}\right) G\left(\theta_{j}\left(\mathbf{p}_{-j}, p_{j}^{\prime}\right)\right)+(1-\lambda)\left(1-p_{j}^{\prime \prime}\right) G\left(\theta_{j}\left(\mathbf{p}_{-j}, p_{j}^{\prime \prime}\right)\right)
\end{gathered}
$$

Multiplying both sides in (A13) with $\lambda G\left(\theta_{j}\left(\mathbf{p}_{-j}, p_{j}^{\prime \prime}\right)\right)+(1-\lambda) G\left(\theta_{j}\left(\mathbf{p}_{-j}, p_{j}^{\prime}\right)\right)$ and rearranging the result we obtain

$$
\begin{array}{r}
\left(1-p_{j}^{\prime}\right) G\left(\theta_{j}\left(\mathbf{p}_{-j}, p_{j}^{\prime}\right)\right)\left[G\left(\theta_{j}\left(\mathbf{p}_{-j}, p_{j}^{\prime \prime}\right)\right)-G\left(\theta_{j}\left(\mathbf{p}_{-j}, p_{j}^{\prime}\right)\right)\right] \\
<\left(1-p_{j}^{\prime \prime}\right) G\left(\theta_{j}\left(\mathbf{p}_{-j}, p_{j}^{\prime \prime}\right)\right)\left[G\left(\theta_{j}\left(\mathbf{p}_{-j}, p_{j}^{\prime \prime}\right)\right)-G\left(\theta_{j}\left(\mathbf{p}_{-j}, p_{j}^{\prime}\right)\right)\right]
\end{array}
$$

But, using the monotonicity properties of Proposition 2 we know that if $p_{j}^{\prime}>p_{j}^{\prime \prime}$ we have that $\theta_{j}\left(\mathbf{p}_{-j}, p_{j}^{\prime \prime}\right)>\theta_{j}\left(\mathbf{p}_{-j}, p_{j}^{\prime}\right)$ and consequently $G\left(\theta_{j}\left(\mathbf{p}_{-j}, p_{j}^{\prime \prime}\right)\right)<G\left(\theta_{j}\left(\mathbf{p}_{-j}, p_{j}^{\prime}\right)\right)$ so that (A14) implies that

$$
\left(1-p_{j}^{\prime}\right) G\left(\theta_{j}\left(\mathbf{p}_{-j}, p_{j}^{\prime}\right)\right)>\left(1-p_{j}^{\prime \prime}\right) G\left(\theta_{j}\left(\mathbf{p}_{-j}, p_{j}^{\prime \prime}\right)\right)
$$

which is inconsistent with the randomization probabilities solving the system (12) and (13) because there must exist some $k \neq j$ such that $\theta_{k}\left(\mathbf{p}_{-j}, p_{j}^{\prime \prime}\right)<\theta_{k}\left(\mathbf{p}_{-j}, p_{j}^{\prime}\right)$ implying that

$$
\left(1-p_{k}\right) G\left(\theta_{k}\left(\mathbf{p}_{-j}, p_{j}^{\prime}\right)\right)<\left(1-p_{k}\right) G\left(\theta_{k}\left(\mathbf{p}_{-j}, p_{j}^{\prime \prime}\right)\right) .
$$

Clearly, (A15) and (A16) are inconsistent with (12) and (13).

\section{A.3 Proof of Proposition 3}

Proof. It is sufficient to show that $\theta_{j}(\mathbf{p}) G\left(\theta_{j}(\mathbf{p})\right)$ is strictly concave as the product of a strictly concave, positive valued and decreasing function and a positive valued increasing concave function is strictly concave. We first note that as $\theta_{j} G\left(\theta_{j}\right)$ is strictly increasing in $\theta_{j}$ and $\theta_{j}(\mathbf{p})$ is strictly concave in $p_{j}$ we have that

$$
\begin{aligned}
& \theta_{j}\left(\mathbf{p}_{-j}, \lambda p_{j}^{\prime}+(1-\lambda) p_{j}^{\prime \prime}\right) G\left(\theta_{j}\left(\mathbf{p}_{-j}, \lambda p_{j}^{\prime}+(1-\lambda) p_{j}^{\prime \prime}\right)\right) \\
&>\quad\left(\lambda \theta_{j}\left(\mathbf{p}_{-j}, p_{j}^{\prime}\right)+(1-\lambda) \theta_{j}\left(\mathbf{p}_{-j}, p_{j}^{\prime \prime}\right)\right) G\left(\lambda \theta_{j}\left(\mathbf{p}_{-j}, p_{j}^{\prime}\right)+(1-\lambda) \theta_{j}\left(\mathbf{p}_{-j}, p_{j}^{\prime \prime}\right)\right)
\end{aligned}
$$

Moreover, $\frac{1}{G}$ being strictly convex implies that

$$
G\left(\lambda \theta_{j}\left(\mathbf{p}_{-j}, p_{j}^{\prime}\right)+(1-\lambda) \theta_{j}\left(\mathbf{p}_{-j}, p_{j}^{\prime \prime}\right)\right)>\frac{G\left(\theta_{j}\left(\mathbf{p}_{-j}, p_{j}^{\prime}\right)\right) G\left(\theta_{j}\left(\mathbf{p}_{-j}, p_{j}^{\prime \prime}\right)\right)}{\lambda G\left(\theta_{j}\left(\mathbf{p}_{-j}, p_{j}^{\prime \prime}\right)\right)+(1-\lambda) G\left(\theta_{j}\left(\mathbf{p}_{-j}, p_{j}^{\prime}\right)\right)}
$$

and combining (A17) and (A18) we have that

$$
\begin{aligned}
& \theta_{j}\left(\mathbf{p}_{-j}, \lambda p_{j}^{\prime}+(1-\lambda) p_{j}^{\prime \prime}\right) G\left(\theta_{j}\left(\mathbf{p}_{-j}, \lambda p_{j}^{\prime}+(1-\lambda) p_{j}^{\prime \prime}\right)\right) \\
> & \frac{\left(\lambda \theta_{j}\left(\mathbf{p}_{-j}, p_{j}^{\prime}\right)+(1-\lambda) \theta_{j}\left(\mathbf{p}_{-j}, p_{j}^{\prime \prime}\right)\right) G\left(\theta_{j}\left(\mathbf{p}_{-j}, p_{j}^{\prime}\right)\right) G\left(\theta_{j}\left(\mathbf{p}_{-j}, p_{j}^{\prime \prime}\right)\right)}{\lambda G\left(\theta_{j}\left(\mathbf{p}_{-j}, p_{j}^{\prime \prime}\right)\right)+(1-\lambda) G\left(\theta_{j}\left(\mathbf{p}_{-j}, p_{j}^{\prime}\right)\right)} \\
> & \lambda \theta_{j}\left(\mathbf{p}_{-j}, p_{j}^{\prime}\right) G\left(\theta_{j}\left(\mathbf{p}_{-j}, p_{j}^{\prime}\right)\right)+(1-\lambda) \theta_{j}\left(\mathbf{p}_{-j}, p_{j}^{\prime \prime}\right) G\left(\theta_{j}\left(\mathbf{p}_{-j}, p_{j}^{\prime \prime}\right)\right),
\end{aligned}
$$


where the second inequality follows because some algebra shows that

$$
\begin{aligned}
& \frac{\left(\lambda \theta_{j}\left(\mathbf{p}_{-j}, p_{j}^{\prime}\right)+(1-\lambda) \theta_{j}\left(\mathbf{p}_{-j}, p_{j}^{\prime \prime}\right)\right) G\left(\theta_{j}\left(\mathbf{p}_{-j}, p_{j}^{\prime}\right)\right) G\left(\theta_{j}\left(\mathbf{p}_{-j}, p_{j}^{\prime \prime}\right)\right)}{\lambda G\left(\theta_{j}\left(\mathbf{p}_{-j}, p_{j}^{\prime \prime}\right)\right)+(1-\lambda) G\left(\theta_{j}\left(\mathbf{p}_{-j}, p_{j}^{\prime}\right)\right)} \\
& -\lambda \theta_{j}\left(\mathbf{p}_{-j}, p_{j}^{\prime}\right) G\left(\theta_{j}\left(\mathbf{p}_{-j}, p_{j}^{\prime}\right)\right)+(1-\lambda) \theta_{j}\left(\mathbf{p}_{-j}, p_{j}^{\prime \prime}\right) G\left(\theta_{j}\left(\mathbf{p}_{-j}, p_{j}^{\prime \prime}\right)\right) \\
= & \lambda(1-\lambda) \frac{\left[\theta_{j}\left(\mathbf{p}_{-j}, p_{j}^{\prime}\right) G\left(\theta_{j}\left(\mathbf{p}_{-j}, p_{j}^{\prime}\right)\right)-\theta_{j}\left(\mathbf{p}_{-j}, p_{j}^{\prime \prime}\right) G\left(\theta_{j}\left(\mathbf{p}_{-j}, p_{j}^{\prime \prime}\right)\right)\left[G\left(\theta_{j}\left(\mathbf{p}_{-j}, p_{j}^{\prime \prime}\right)\right)-G\left(\theta_{j}\left(\mathbf{p}_{-j}, p_{j}^{\prime \prime}\right)\right)\right]\right.}{\lambda G\left(\theta_{j}\left(\mathbf{p}_{-j}, p_{j}^{\prime \prime}\right)\right)+(1-\lambda) G\left(\theta_{j}\left(\mathbf{p}_{-j}, p_{j}^{\prime}\right)\right)}>0
\end{aligned}
$$

where the inequality holds because $\theta_{j} G\left(\theta_{j}\right)$ and $G\left(\theta_{j}\right)$ are both strictly decreasing in $\theta_{j}$.

\section{A.4 Proof of Proposition 4}

Proof. By differentiation of the profit function in (14)

$$
\begin{aligned}
\frac{1}{n} \frac{\partial \pi_{j}(\boldsymbol{p})}{\partial p_{i} \partial p_{j}}= & \frac{d h_{j}\left(p_{j}\right)}{d p_{j}} \frac{\partial \theta_{j}(\boldsymbol{p})}{\partial p_{i}}\left[G\left(\theta_{j}(\boldsymbol{p})\right)+\theta_{j}(\boldsymbol{p}) G^{\prime}\left(\theta_{j}(\boldsymbol{p})\right)\right] \\
& +h_{j}\left(p_{j}\right) \frac{\partial^{2} \theta_{j}(\boldsymbol{p})}{\partial p_{i} \partial p_{j}}\left[G\left(\theta_{j}(\boldsymbol{p})\right)+\theta_{j}(\boldsymbol{p}) G^{\prime}\left(\theta_{j}(\boldsymbol{p})\right)\right] \\
& +h_{j}\left(p_{j}\right) \frac{\partial \theta_{j}(\boldsymbol{p})}{\partial p_{i}} \frac{\partial \theta_{j}(\boldsymbol{p})}{\partial p_{j}}\left[2 G^{\prime}\left(\theta_{j}(\boldsymbol{p})\right)+\theta_{j}(\boldsymbol{p}) G^{\prime \prime}\left(\theta_{j}(\boldsymbol{p})\right)\right]
\end{aligned}
$$

We note that strict convexity of $1 / G$ and the fact that $G$ takes on only positive values implies that $G^{\prime \prime}(\theta)<2\left[G^{\prime}(\theta)\right]^{2} / G(\theta)$, which combined with (A21) implies that

$$
\begin{aligned}
\frac{1}{n} \frac{\partial \pi_{j}(\boldsymbol{p})}{\partial p_{i} \partial p_{j}}> & \frac{d h_{j}\left(p_{j}\right)}{d p_{j}} \frac{\partial \theta_{j}(\boldsymbol{p})}{\partial p_{i}}\left[G\left(\theta_{j}(\boldsymbol{p})\right)+\theta_{j}(\boldsymbol{p}) G^{\prime}\left(\theta_{j}(\boldsymbol{p})\right)\right] \\
& +h_{j}\left(p_{j}\right) \frac{\partial^{2} \theta_{j}(\boldsymbol{p})}{\partial p_{i} \partial p_{j}}\left[G\left(\theta_{j}(\boldsymbol{p})\right)+\theta_{j}(\boldsymbol{p}) G^{\prime}\left(\theta_{j}(\boldsymbol{p})\right)\right] \\
& +h_{j}\left(p_{j}\right) \frac{\partial \theta_{j}(\boldsymbol{p})}{\partial p_{i}} \frac{\partial \theta_{j}(\boldsymbol{p})}{\partial p_{j}} \frac{2 G^{\prime}\left(\theta_{j}(\boldsymbol{p})\right)}{G\left(\theta_{j}(\boldsymbol{p})\right)}\left[G\left(\theta_{j}(\boldsymbol{p})\right)+\theta_{j}(\boldsymbol{p}) G^{\prime}\left(\theta_{j}(\boldsymbol{p})\right)\right]
\end{aligned}
$$

Since $\theta G(\theta)$ is strictly increasing we obtain a sufficient condition for $\frac{\partial \pi_{j}(\boldsymbol{p})}{\partial p_{i} \partial p_{j}}>0$ given by

$$
\frac{d h_{j}\left(p_{j}\right)}{d p_{j}} \frac{\partial \theta_{j}(\boldsymbol{p})}{\partial p_{i}}+h_{j}\left(p_{j}\right) \frac{\partial^{2} \theta_{j}(\boldsymbol{p})}{\partial p_{i} \partial p_{j}}+h_{j}\left(p_{j}\right) \frac{\partial \theta_{j}(\boldsymbol{p})}{\partial p_{i}} \frac{\partial \theta_{j}(\boldsymbol{p})}{\partial p_{j}} \frac{2 G^{\prime}\left(\theta_{j}(\boldsymbol{p})\right)}{G\left(\theta_{j}(\boldsymbol{p})\right)} \geq 0
$$

where the first and the third term are strictly positive and the middle term is ambiguous. Reversing the roles of $i$ and $j$ in (17), differentiating the result and simplifying slightly we can express the cross derivative $\frac{\partial^{2} \theta_{j}(\boldsymbol{p})}{\partial p_{i} \partial p_{j}}$ as

$$
\begin{aligned}
\frac{\partial^{2} \theta_{j}(\mathbf{p})}{\partial p_{i} \partial p_{j}}= & -\frac{d}{d p_{j}}\left[\frac{G\left(\theta_{i}(\mathbf{p})\right)}{\left(1-p_{i}\right) G^{\prime}\left(\theta_{i}(\mathbf{p})\right)}\right]\left[\frac{\frac{G\left(\theta_{j}(\mathbf{p})\right)}{G^{\prime}\left(\theta_{j}(\mathbf{p})\right)}}{\left.\sum_{k=1}^{s} \frac{G\left(\theta_{k}(\mathbf{p})\right)}{G^{\prime}\left(\theta_{k}(\mathbf{p})\right)}\right]}\right. \\
& -\frac{G\left(\theta_{i}(\mathbf{p})\right)}{\left(1-p_{i}\right) G^{\prime}\left(\theta_{i}(\mathbf{p})\right)} \frac{\frac{d}{d p_{j}}\left[\frac{G\left(\theta_{j}(\mathbf{p})\right)}{G^{\prime}\left(\theta_{j}(\mathbf{p})\right)}\right] \sum_{k \neq j} \frac{G\left(\theta_{k}(\mathbf{p})\right)}{G^{\prime}\left(\theta_{k}(\mathbf{p})\right)}-\left[\frac{G\left(\theta_{j}(\mathbf{p})\right)}{G^{\prime}\left(\theta_{j}(\mathbf{p})\right)}\right] \sum_{k \neq j} \frac{d}{d p_{j}} \frac{G\left(\theta_{k}(\mathbf{p})\right)}{G^{\prime}\left(\theta_{k}(\mathbf{p})\right)}}{\left[\sum_{k=1}^{s} \frac{G\left(\theta_{k}(\mathbf{p})\right)}{G^{\prime}\left(\theta_{k}(\mathbf{p})\right)}\right]^{2}},
\end{aligned}
$$


where strict convexity of $G$ and $1 / G$ implies that

$$
\frac{d}{d p_{j}} \frac{G\left(\theta_{k}(\mathbf{p})\right)}{G^{\prime}\left(\theta_{k}(\mathbf{p})\right)} \in\left(-\left|\frac{\partial \theta_{k}(\boldsymbol{p})}{\partial p_{j}}\right|,\left|\frac{\partial \theta_{k}(\boldsymbol{p})}{\partial p_{j}}\right|\right)
$$

for every $k$ where $\left|\frac{\partial \theta_{k}(\boldsymbol{p})}{\partial p_{j}}\right|$ denotes the absolute value. It follows that

$$
\begin{aligned}
& \left|\frac{d}{d p_{j}}\left[\frac{G\left(\theta_{j}(\mathbf{p})\right)}{G^{\prime}\left(\theta_{j}(\mathbf{p})\right)}\right] \sum_{k \neq j} \frac{G\left(\theta_{k}(\mathbf{p})\right)}{G^{\prime}\left(\theta_{k}(\mathbf{p})\right)}-\left[\frac{G\left(\theta_{j}(\mathbf{p})\right)}{G^{\prime}\left(\theta_{j}(\mathbf{p})\right)}\right] \sum_{k \neq j} \frac{d}{d p_{j}} \frac{G\left(\theta_{k}(\mathbf{p})\right)}{G^{\prime}\left(\theta_{k}(\mathbf{p})\right)}\right| \\
> & \frac{\partial \theta_{j}(\boldsymbol{p})}{\partial p_{j}} \sum_{k \neq j} \frac{G\left(\theta_{k}(\mathbf{p})\right)}{G^{\prime}\left(\theta_{k}(\mathbf{p})\right)}-\left[\frac{G\left(\theta_{j}(\mathbf{p})\right)}{G^{\prime}\left(\theta_{j}(\mathbf{p})\right)}\right] \sum_{k \neq j} \frac{\partial \theta_{k}(\boldsymbol{p})}{\partial p_{j}}=\frac{\partial \theta_{j}(\boldsymbol{p})}{\partial p_{j}} \sum_{k=1}^{s} \frac{G\left(\theta_{k}(\mathbf{p})\right)}{G^{\prime}\left(\theta_{k}(\mathbf{p})\right)},
\end{aligned}
$$

and combining (A25) and (A26) with (A24) we have that

$$
\begin{aligned}
\frac{\partial^{2} \theta_{j}(\boldsymbol{p})}{\partial p_{i} \partial p_{j}} & >-\frac{1}{1-p_{i}}\left[\frac{\frac{G\left(\theta_{j}(\mathbf{p})\right)}{G^{\prime}\left(\theta_{j}(\mathbf{p})\right)}}{\sum_{k=1}^{s} \frac{G\left(\theta_{k}(\mathbf{p})\right)}{G^{\prime}\left(\theta_{k}(\mathbf{p})\right)}}\right] \frac{\partial \theta_{i}(\boldsymbol{p})}{\partial p_{j}}+\frac{G\left(\theta_{i}(\mathbf{p})\right)}{\left(1-p_{i}\right) G^{\prime}\left(\theta_{i}(\mathbf{p})\right)} \frac{\frac{\partial \theta_{j}(\boldsymbol{p})}{\partial p_{j}}}{\sum_{k=1}^{s} \frac{G\left(\theta_{k}(\mathbf{p})\right)}{G^{\prime}\left(\theta_{k}(\mathbf{p})\right)}} \\
& =\frac{G^{\prime}\left(\theta_{i}(\mathbf{p})\right)}{G\left(\theta_{i}(\mathbf{p})\right)} \frac{\partial \theta_{j}(\boldsymbol{p})}{\partial p_{i}} \frac{\partial \theta_{i}(\boldsymbol{p})}{\partial p_{j}}-\frac{G^{\prime}\left(\theta_{j}(\mathbf{p})\right)}{G\left(\theta_{j}(\mathbf{p})\right)} \frac{\partial \theta_{j}(\boldsymbol{p})}{\partial p_{i}} \frac{\partial \theta_{j}(\boldsymbol{p})}{\partial p_{j}},
\end{aligned}
$$

which combined with (A23) implies that a sufficient condition for $\frac{\partial \pi_{j}(\boldsymbol{p})}{\partial p_{i} \partial p_{j}}>0$ is that

$$
\frac{d h_{j}\left(p_{j}\right)}{d p_{j}}+h_{j}\left(p_{j}\right) \frac{G^{\prime}\left(\theta_{i}(\mathbf{p})\right)}{G\left(\theta_{i}(\mathbf{p})\right)} \frac{\partial \theta_{i}(\boldsymbol{p})}{\partial p_{j}}+h_{j}\left(p_{j}\right) \frac{G^{\prime}\left(\theta_{j}(\boldsymbol{p})\right)}{G\left(\theta_{j}(\boldsymbol{p})\right)} \frac{\partial \theta_{j}(\boldsymbol{p})}{\partial p_{j}} \geq 0,
$$

but substituting from (16) and (17) we have that

$$
\begin{aligned}
& \frac{d h_{j}\left(p_{j}\right)}{d p_{j}}+h_{j}\left(p_{j}\right) \frac{G^{\prime}\left(\theta_{i}(\mathbf{p})\right)}{G\left(\theta_{i}(\mathbf{p})\right)} \frac{\partial \theta_{i}(\boldsymbol{p})}{\partial p_{j}}+h_{j}\left(p_{j}\right) \frac{G^{\prime}\left(\theta_{j}(\boldsymbol{p})\right)}{G\left(\theta_{j}(\boldsymbol{p})\right)} \frac{\partial \theta_{j}(\boldsymbol{p})}{\partial p_{j}} \\
= & \frac{d h_{j}\left(p_{j}\right)}{d p_{j}}+\frac{h_{j}\left(p_{j}\right)}{\left(1-p_{j}\right)}\left[\frac{\sum_{k \neq j} \frac{G\left(\theta_{k}(\mathbf{p})\right)}{G^{\prime}\left(\theta_{k}(\mathbf{p})\right)}-\frac{G\left(\theta_{j}(\mathbf{p})\right)}{G^{\prime}\left(\theta_{j}(\mathbf{p})\right)}}{\sum_{k=1}^{s} \frac{G\left(\theta_{k}(\mathbf{p})\right)}{G^{\prime}\left(\theta_{k}(\mathbf{p})\right)}}\right]>0,
\end{aligned}
$$

where the inequality in (A29) follows because we know that $\frac{\partial \theta_{j}(\mathbf{p})}{\partial p_{j}}<0$ and $\frac{\partial \theta_{i}(\boldsymbol{p})}{\partial p_{j}}>0$ for every $i \neq j$ which requires that

$$
\frac{\partial \theta_{j}(\mathbf{p})}{\partial p_{j}}-\frac{\partial \theta_{i}(\mathbf{p})}{\partial p_{j}}=\frac{G\left(\theta_{j}(\mathbf{p})\right)}{\left(1-p_{j}\right) G^{\prime}\left(\theta_{j}(\mathbf{p})\right)} \frac{\left[\sum_{k \neq j} \frac{G\left(\theta_{k}(\mathbf{p})\right)}{G^{\prime}\left(\theta_{k}(\mathbf{p})\right)}-\frac{G\left(\theta_{i}(\mathbf{p})\right)}{G^{\prime}\left(\theta_{i}(\mathbf{p})\right)}\right]}{\sum_{i=1}^{s} \frac{G\left(\theta_{i}(\mathbf{p})\right)}{G^{\prime}\left(\theta_{i}(\mathbf{p})\right)}}<0
$$

as otherwise $0=\frac{\partial \theta_{j}(\mathbf{p})}{\partial p_{j}}+\sum_{i \neq j} \frac{\partial \theta_{i}(\boldsymbol{p})}{\partial p_{j}}>\frac{\partial \theta_{j}(\mathbf{p})}{\partial p_{j}}+\frac{\partial \theta_{i}(\mathbf{p})}{\partial p_{j}} \geq 0$. Hence, if the inequality in (A29) fails we have that

$$
\sum_{k \neq j} \frac{G\left(\theta_{k}(\mathbf{p})\right)}{G^{\prime}\left(\theta_{k}(\mathbf{p})\right)}-\frac{G\left(\theta_{i}(\mathbf{p})\right)}{G^{\prime}\left(\theta_{i}(\mathbf{p})\right)}<0<\sum_{k \neq j} \frac{G\left(\theta_{k}(\mathbf{p})\right)}{G^{\prime}\left(\theta_{k}(\mathbf{p})\right)}-\frac{G\left(\theta_{j}(\mathbf{p})\right)}{G^{\prime}\left(\theta_{j}(\mathbf{p})\right)}
$$

so that $\frac{G\left(\theta_{j}(\mathbf{p})\right)}{G^{\prime}\left(\theta_{j}(\mathbf{p})\right)}<\frac{G\left(\theta_{i}(\mathbf{p})\right)}{G^{\prime}\left(\theta_{i}(\mathbf{p})\right)}<\dot{0}$. But, in this case we observe that $\frac{\partial \theta_{j}(\mathbf{p})}{\partial p_{j}}+\frac{\partial \theta_{i}(\mathbf{p})}{\partial p_{j}}<0$ implying that $\frac{G^{\prime}\left(\theta_{i}(\mathbf{p})\right)}{G\left(\theta_{i}(\mathbf{p})\right)} \frac{\partial \theta_{i}(\boldsymbol{p})}{\partial p_{j}}+\frac{G^{\prime}\left(\theta_{j}(\boldsymbol{p})\right)}{G\left(\theta_{j}(\boldsymbol{p})\right)} \frac{\partial \theta_{j}(\boldsymbol{p})}{\partial p_{j}}>0$, which completes the proof. 


\section{A.5 Proof of Proposition 6}

As a preliminary step one notes:

Lemma A1 Suppose that $G(\theta) \geq 0$ for each $\theta$ and strictly decreasing, $\theta G(\theta)$ is strictly increasing, and that $1 / G(\theta)$ is strictly convex. Then, $\theta G(\theta)$ is strictly concave.

Proof. Strict convexity of $\frac{1}{G(\theta)}$ means that

$$
\frac{1}{G\left(\lambda \theta^{\prime}+(1-\lambda) \theta^{\prime \prime}\right)}<\frac{\lambda}{G\left(\theta^{\prime}\right)}+\frac{1-\lambda}{G\left(\theta^{\prime \prime}\right)}
$$

holds for each distinct pair $\left(\theta^{\prime}, \theta^{\prime \prime}\right)$ and every $\lambda \in(0,1)$. This can be rearranged as

$$
G\left(\lambda \theta^{\prime}+(1-\lambda) \theta^{\prime \prime}\right)>\frac{G\left(\theta^{\prime \prime}\right) G\left(\theta^{\prime}\right)}{\lambda G\left(\theta^{\prime \prime}\right)+(1-\lambda) G\left(\theta^{\prime}\right)}
$$

because $G$ takes on only positive values. Hence

$$
\begin{aligned}
& \left(\lambda \theta^{\prime}+(1-\lambda) \theta^{\prime \prime}\right) G\left(\lambda \theta^{\prime}+(1-\lambda) \theta^{\prime \prime}\right)-\lambda \theta^{\prime} G\left(\theta^{\prime}\right)-(1-\lambda) \theta^{\prime \prime} G\left(\theta^{\prime \prime \prime}\right) \\
> & \left(\lambda \theta^{\prime}+(1-\lambda) \theta^{\prime \prime}\right) \frac{G\left(\theta^{\prime \prime}\right) G\left(\theta^{\prime}\right)}{\lambda G\left(\theta^{\prime \prime}\right)+(1-\lambda) G\left(\theta^{\prime}\right)}-\lambda \theta^{\prime} G\left(\theta^{\prime}\right)-(1-\lambda) \theta^{\prime \prime} G\left(\theta^{\prime \prime \prime}\right) \\
= & \lambda \theta^{\prime} G\left(\theta^{\prime}\right)\left[\frac{G\left(\theta^{\prime \prime}\right)}{\lambda G\left(\theta^{\prime \prime}\right)+(1-\lambda) G\left(\theta^{\prime}\right)}-1\right]+(1-\lambda) \theta^{\prime \prime} G\left(\theta^{\prime \prime \prime}\right)\left[\frac{G\left(\theta^{\prime}\right)}{\lambda G\left(\theta^{\prime \prime}\right)+(1-\lambda) G\left(\theta^{\prime}\right)}-1\right] \\
= & \lambda(1-\lambda) \theta^{\prime} G\left(\theta^{\prime}\right)\left[\frac{G\left(\theta^{\prime \prime}\right)-G\left(\theta^{\prime}\right)}{\lambda G\left(\theta^{\prime \prime}\right)+(1-\lambda) G\left(\theta^{\prime}\right)}\right]+(1-\lambda) \lambda \theta^{\prime \prime} G\left(\theta^{\prime \prime \prime}\right)\left[\frac{G\left(\theta^{\prime}\right)-G\left(\theta^{\prime \prime}\right)}{\lambda G\left(\theta^{\prime \prime}\right)+(1-\lambda) G\left(\theta^{\prime}\right)}\right] \\
= & \lambda(1-\lambda)\left[\frac{G\left(\theta^{\prime \prime}\right)-G\left(\theta^{\prime}\right)}{\lambda G\left(\theta^{\prime \prime}\right)+(1-\lambda) G\left(\theta^{\prime}\right)}\right]\left[\theta^{\prime} G\left(\theta^{\prime}\right)-\theta^{\prime \prime} G\left(\theta^{\prime \prime \prime}\right)\right]>0
\end{aligned}
$$

as $G\left(\theta^{\prime \prime}\right)-G\left(\theta^{\prime}\right)$ and $\theta^{\prime} G\left(\theta^{\prime}\right)-\theta^{\prime \prime} G\left(\theta^{\prime \prime \prime}\right)$ have the same sign as $G$ is strictly decreasing and $\theta G(\theta)$ is strictly increasing.

Lemma A2 Suppose that $\mathbf{p}^{*}$ and $\mathbf{p}^{* *}$ with $p^{*}<<p^{* *}$. Then there is at least one $j$ such that $\theta_{j}\left(\boldsymbol{p}^{*}\right)>\theta_{j}\left(\boldsymbol{p}^{* *}\right)$.

Proof. If not we have that $\theta_{j}\left(\boldsymbol{p}^{*}\right)=\theta_{j}\left(\boldsymbol{p}^{* *}\right)$ for each $j$. Evaluating the first order condition at $\mathbf{p}^{*}$ and $\mathbf{p}^{* *}$ we can make the same argument as when ruling out multiple symmetric equilibria in (19).

Lemma A3 Suppose that $\mathbf{p}^{*}$ and $\mathbf{p}^{* *}$ with $p^{*}<<p^{* *}$ and $\theta_{j}\left(\boldsymbol{p}^{*}\right)>\theta_{j}\left(\boldsymbol{p}^{* *}\right)$. Then, $\left|\frac{\partial \theta_{j}\left(\boldsymbol{p}^{*}\right)}{\partial p_{j}}\right|>$ $\left|\frac{\partial \theta_{j}\left(\boldsymbol{p}^{* *}\right)}{\partial p_{j}}\right|$

Proof. Then first order condition.

$$
0=h_{j}^{\prime}\left(p_{j}\right) \theta_{j}(\boldsymbol{p}) G\left(\theta_{j}(\boldsymbol{p})\right)+h_{j}\left(p_{j}\right)\left[G\left(\theta_{j}(\boldsymbol{p})\right)+\theta_{j}(\boldsymbol{p}) G^{\prime}\left(\theta_{j}(\boldsymbol{p})\right)\right] \frac{\partial \theta_{j}(\boldsymbol{p})}{\partial p_{j}}
$$


must hold evaluated at both $\mathbf{p}^{*}$ and $\mathbf{p}^{* *}$. We have that $p^{*}<<p^{* *}$, so $h_{j}^{\prime}\left(p_{j}^{*}\right) \geq h_{j}^{\prime}\left(p_{j}^{* *}\right)>0$ because $h_{j}$ is concave and increasing. Also $\theta_{j}\left(\boldsymbol{p}^{*}\right)>\theta_{j}\left(\boldsymbol{p}^{* *}\right)$, so $\theta_{j}\left(\boldsymbol{p}^{*}\right) G\left(\theta_{j}\left(\boldsymbol{p}^{*}\right)\right)>\theta_{j}\left(\boldsymbol{p}^{* *}\right) G\left(\theta_{j}\left(\boldsymbol{p}^{* *}\right)\right)$ as $\theta G(\theta)$ is strictly increasing. Hence

$$
h_{j}^{\prime}\left(p_{j}^{*}\right) \theta_{j}\left(\boldsymbol{p}^{*}\right) G\left(\theta_{j}\left(\boldsymbol{p}^{*}\right)\right)>h_{j}\left(p_{j}^{* *}\right) \theta_{j}\left(\boldsymbol{p}^{* *}\right) G\left(\theta_{j}\left(\boldsymbol{p}^{* *}\right)\right)>0 .
$$

Next, $h_{j}\left(p_{j}^{*}\right)<h_{j}\left(p_{j}^{* *}\right)$ as $h_{j}$ is increasing and

$$
\begin{aligned}
0 & <\left.\frac{d}{d \theta}\right|_{\theta_{j}\left(\boldsymbol{p}^{*}\right)} \theta G(\theta)=G\left(\theta_{j}\left(\boldsymbol{p}^{*}\right)\right)+\theta_{j}\left(\boldsymbol{p}^{*}\right) G^{\prime}\left(\theta_{j}\left(\boldsymbol{p}^{*}\right)\right) \\
& <G\left(\theta_{j}\left(\boldsymbol{p}^{* *}\right)\right)+\theta_{j}\left(\boldsymbol{p}^{* *}\right) G^{\prime}\left(\theta_{j}\left(\boldsymbol{p}^{* *}\right)\right)=\left.\frac{d}{d \theta}\right|_{\theta_{j}\left(\boldsymbol{p}^{* *}\right)} \theta G(\theta)
\end{aligned}
$$

because $\theta G(\theta)$ is increasing and strictly concave from Lemma A1. Combining (A33) and (A34) it follows that the only way (A32) can hold evaluated at both $\mathbf{p}^{*}$ and $\mathbf{p}^{* *}$ is if $\left|\frac{\partial \theta_{j}\left(\boldsymbol{p}^{*}\right)}{\partial p_{j}}\right|>\left|\frac{\partial \theta_{j}\left(\boldsymbol{p}^{* *}\right)}{\partial p_{j}}\right|$.

To finish the proof of Proposition 6 we first note that

$$
\left|\frac{\partial \theta_{j}(\boldsymbol{p})}{\partial p_{j}}\right|=\left|\frac{G\left(\theta_{j}(\boldsymbol{p})\right)}{\left(1-p_{j}\right) G^{\prime}\left(\theta_{j}(\boldsymbol{p})\right)}\right| \frac{\sum_{i \neq j} \frac{G\left(\theta_{i}(\boldsymbol{p})\right)}{G^{\prime}\left(\theta_{i}(\boldsymbol{p})\right)}}{\sum_{i=1}^{s} \frac{G\left(\theta_{i}(\boldsymbol{p})\right)}{G^{\prime}\left(\theta_{i}(\boldsymbol{p})\right)}}=\left|\frac{\left[G\left(\theta_{j}(\boldsymbol{p})\right)\right]^{2}}{U(\boldsymbol{p}) G^{\prime}\left(\theta_{j}(\boldsymbol{p})\right)}\right| \frac{\sum_{i \neq j} \frac{G\left(\theta_{i}(\boldsymbol{p})\right)}{G^{\prime}\left(\theta_{i}(\boldsymbol{p})\right)}}{\sum_{i=1}^{s} \frac{G\left(\theta_{i}(\boldsymbol{p})\right)}{G^{\prime}\left(\theta_{i}(\boldsymbol{p})\right)}},
$$

where $U(\boldsymbol{p})=\left(1-p_{j}\right) G^{\prime}\left(\theta_{j}(\boldsymbol{p})\right)$ is the equilibrium utility of the buyer. Obviously, $U\left(\boldsymbol{p}^{*}\right)>$ $U\left(\mathbf{p}^{* *}\right)>0$ and strict convexity of $\frac{1}{G}$ means that

$$
\frac{d}{d \theta} \frac{1}{G(\theta)}=-\frac{G^{\prime}(\theta)}{[G(\theta)]^{2}}=\left|\frac{G^{\prime}(\theta)}{[G(\theta)]^{2}}\right|
$$

is increasing in $\theta$. As $\theta_{j}\left(\boldsymbol{p}^{*}\right)>\theta_{j}\left(\boldsymbol{p}^{* *}\right)$ we conclude that $\left|\frac{\left[G\left(\theta_{j}\left(\boldsymbol{p}^{*}\right)\right)\right]^{2}}{G^{\prime}\left(\theta_{j}\left(\boldsymbol{p}^{*}\right)\right)}\right|<\left|\frac{\left[G\left(\theta_{j}\left(\boldsymbol{p}^{* *}\right)\right]^{2}\right.}{G^{\prime}\left(\theta_{j}\left(\boldsymbol{p}^{* *}\right)\right)}\right|$. Combined this means that

$$
\left|\frac{\left[G\left(\theta_{j}\left(\boldsymbol{p}^{*}\right)\right)\right]^{2}}{U\left(\boldsymbol{p}^{*}\right) G^{\prime}\left(\theta_{j}\left(\boldsymbol{p}^{*}\right)\right)}\right|<\left|\frac{\left[G\left(\theta_{j}\left(\boldsymbol{p}^{* *}\right)\right)\right]^{2}}{U\left(\boldsymbol{p}^{* *}\right) G^{\prime}\left(\theta_{j}\left(\boldsymbol{p}^{* *}\right)\right)}\right|
$$

so for $\left|\frac{\partial \theta_{j}\left(\boldsymbol{p}^{*}\right)}{\partial p_{j}}\right|>\left|\frac{\partial \theta_{j}\left(\boldsymbol{p}^{* *}\right)}{\partial p_{j}}\right|$ it must be that

$$
\frac{\sum_{i \neq j} \frac{G\left(\theta_{i}\left(\boldsymbol{p}^{*}\right)\right)}{G^{\prime}\left(\theta_{i}\left(\boldsymbol{p}^{*}\right)\right)}}{\sum_{i=1}^{s} \frac{G\left(\theta_{i}\left(\boldsymbol{p}^{*}\right)\right)}{G^{\prime}\left(\theta_{i}\left(\boldsymbol{p}^{*}\right)\right)}}>\frac{\sum_{i \neq j} \frac{G\left(\theta_{i}\left(\boldsymbol{p}^{* *}\right)\right)}{G^{\prime}\left(\theta_{i}\left(\boldsymbol{p}^{* *}\right)\right)}}{\sum_{i=1}^{s} \frac{G\left(\theta_{i}\left(\boldsymbol{p}^{* *}\right)\right)}{G^{\prime}\left(\theta_{i}\left(\boldsymbol{p}^{* *}\right)\right)}}
$$

where we note that the buyer indifference conditions imply that

$$
\frac{\sum_{i \neq j} \frac{G\left(\theta_{i}(\boldsymbol{p})\right)}{G^{\prime}\left(\theta_{i}(\boldsymbol{p})\right)}}{\sum_{i=1}^{s} \frac{G\left(\theta_{i}(\boldsymbol{p})\right)}{G^{\prime}\left(\theta_{i}(\boldsymbol{p})\right)}}=\frac{\sum_{i \neq j} \frac{\left(1-p_{i}^{*}\right) G\left(\theta_{i}(\boldsymbol{p})\right)}{\left(1-p_{i}^{*}\right) G^{\prime}\left(\theta_{i}(\boldsymbol{p})\right)}}{\sum_{i=1}^{s} \frac{\left(1-p_{i}^{*}\right) G\left(\theta_{i}(\boldsymbol{p})\right)}{\left(1-p_{i}^{*}\right) G^{\prime}\left(\theta_{i}(\boldsymbol{p})\right)}}=\frac{\sum_{i \neq j} \frac{1}{\left(1-p_{i}^{*}\right) G^{\prime}\left(\theta_{i}(\boldsymbol{p})\right)}}{\sum_{i=1}^{s} \frac{1}{\left(1-p_{i}^{*}\right) G^{\prime}\left(\theta_{i}(\boldsymbol{p})\right)}}=1-\frac{\frac{1}{\left(1-p_{j}^{*}\right) G^{\prime}\left(\theta_{j}(\boldsymbol{p})\right)}}{\sum_{i=1}^{s} \frac{1}{\left(1-p_{i}^{*}\right) G^{\prime}\left(\theta_{i}(\boldsymbol{p})\right)}}
$$

Hence, multiplicity is only possible if

$$
\frac{\frac{1}{\left(1-p_{j}^{*}\right) G^{\prime}\left(\theta_{j}\left(\boldsymbol{p}^{*}\right)\right)}}{\sum_{i=1}^{s} \frac{1}{\left(1-p_{i}^{*}\right) G^{\prime}\left(\theta_{i}\left(\boldsymbol{p}^{*}\right)\right)}}<\frac{\frac{1}{\left(1-p_{j}^{* *}\right) G^{\prime}\left(\theta_{j}\left(\boldsymbol{p}^{* *}\right)\right)}}{\sum_{i=1}^{s} \frac{1}{\left(1-p_{i}^{* *}\right) G^{\prime}\left(\theta_{i}\left(\boldsymbol{p}^{* *}\right)\right)}} .
$$


holds for every $j$ such that $\theta_{j}\left(\boldsymbol{p}^{*}\right)>\theta_{j}\left(\boldsymbol{p}^{* *}\right)$, implying that there is some $k$ such that $\theta_{k}\left(\boldsymbol{p}^{*}\right) \leq$ $\theta_{k}\left(\boldsymbol{p}^{* *}\right)$ such that

$$
\frac{\frac{1}{\left(1-p_{k}^{*}\right) G^{\prime}\left(\theta_{k}\left(\boldsymbol{p}^{*}\right)\right)}}{\sum_{i=1}^{s} \frac{1}{\left(1-p_{i}^{*}\right) G^{\prime}\left(\theta_{i}\left(\boldsymbol{p}^{*}\right)\right)}}>\frac{\frac{1}{\left(1-p_{k}^{* *}\right) G^{\prime}\left(\theta_{k}\left(\boldsymbol{p}^{* *}\right)\right)}}{\sum_{i=1}^{s} \frac{1}{\left(1-p_{i}^{* *}\right) G^{\prime}\left(\theta_{i}\left(\boldsymbol{p}^{* *}\right)\right)}},
$$

where we know that $\theta_{k}\left(\boldsymbol{p}^{*}\right) \leq \theta_{k}\left(\boldsymbol{p}^{* *}\right) \Rightarrow G^{\prime}\left(\theta_{k}\left(\boldsymbol{p}^{*}\right)\right) \leq G^{\prime}\left(\theta_{k}\left(\boldsymbol{p}^{* *}\right)\right) \Rightarrow\left|G^{\prime}\left(\theta_{k}\left(\boldsymbol{p}^{*}\right)\right)\right| \geq\left|G^{\prime}\left(\theta_{k}\left(\boldsymbol{p}^{* *}\right)\right)\right|$, so

$$
\left|\frac{1}{\left(1-p_{k}^{*}\right) G^{\prime}\left(\theta_{k}\left(\boldsymbol{p}^{*}\right)\right)}\right|<\left|\frac{1}{\left(1-p_{k}^{* *}\right) G^{\prime}\left(\theta_{k}\left(\boldsymbol{p}^{* *}\right)\right)}\right|
$$

Hence

$$
\left|\sum_{i=1}^{s} \frac{1}{\left(1-p_{i}^{*}\right) G^{\prime}\left(\theta_{i}\left(\boldsymbol{p}^{*}\right)\right)}\right|<\sum_{i=1}^{s} \frac{1}{\left(1-p_{i}^{* *}\right) G^{\prime}\left(\theta_{i}\left(\boldsymbol{p}^{* *}\right)\right)}
$$

and it follows that from (A36)

$$
\left|\frac{1}{\left(1-p_{j}^{*}\right) G^{\prime}\left(\theta_{j}\left(\boldsymbol{p}^{*}\right)\right)}\right|<\left|\frac{1}{\left(1-p_{j}^{* *}\right) G^{\prime}\left(\theta_{j}\left(\boldsymbol{p}^{* *}\right)\right)}\right|
$$

holds for every $j$ such that $\theta_{j}\left(\boldsymbol{p}^{*}\right)>\theta_{j}\left(\boldsymbol{p}^{* *}\right)$. But, rewriting the derivative in (A35) as

$$
\begin{aligned}
\left|\frac{\partial \theta_{j}\left(\boldsymbol{p}^{*}\right)}{\partial p_{j}}\right| & =\left|\frac{G\left(\theta_{j}\left(\boldsymbol{p}^{*}\right)\right)}{\left(1-p_{j}^{*}\right) G^{\prime}\left(\theta_{j}\left(\boldsymbol{p}^{*}\right)\right)}\right| \frac{\sum_{i \neq j} \frac{G\left(\theta_{i}\left(\boldsymbol{p}^{*}\right)\right)}{G^{\prime}\left(\theta_{i}\left(\boldsymbol{p}^{*}\right)\right)}}{\sum_{i=1}^{s} \frac{G\left(\theta_{i}\left(\boldsymbol{p}^{*}\right)\right)}{G^{\prime}\left(\theta_{i}\left(\boldsymbol{p}^{*}\right)\right)}} \\
& =\left|\frac{G\left(\theta_{j}\left(\boldsymbol{p}^{*}\right)\right)}{\left(1-p_{j}^{*}\right) G^{\prime}\left(\theta_{j}\left(\boldsymbol{p}^{*}\right)\right)}\right| \frac{\sum_{i \neq j} \frac{1}{\left(1-p_{i}^{*}\right) G^{\prime}\left(\theta_{i}\left(\boldsymbol{p}^{* *}\right)\right)}}{\sum_{i=1}^{s} \frac{1}{\left(1-p_{i}^{*}\right) G^{\prime}\left(\theta_{i}\left(\boldsymbol{p}^{* *}\right)\right)}} \\
& <\left|\frac{G\left(\theta_{j}\left(\boldsymbol{p}^{*}\right)\right)}{\left(1-p_{j}^{*}\right) G^{\prime}\left(\theta_{j}\left(\boldsymbol{p}^{*}\right)\right)}\right| \frac{\sum_{i \neq j} \frac{1}{\left(1-p_{i}^{* *}\right) G^{\prime}\left(\theta_{i}\left(\boldsymbol{p}^{* *}\right)\right)}}{\sum_{i=1}^{s} \frac{1}{\left(1-p_{i}^{*}\right) G^{\prime}\left(\theta_{i}\left(\boldsymbol{p}^{* *}\right)\right)}} .
\end{aligned}
$$

where we use that (A38) is true for all $k$ such that $\theta_{k}\left(\boldsymbol{p}^{*}\right) \leq \theta_{k}\left(\boldsymbol{p}^{* *}\right)$ and (A40) is true for all $j$ such that $\theta_{j}\left(\boldsymbol{p}^{*}\right)>\theta_{j}\left(\boldsymbol{p}^{* *}\right)$. But, again using the inequality in (A36) we find that

$$
\begin{aligned}
\left|\frac{\partial \theta_{j}\left(\boldsymbol{p}^{*}\right)}{\partial p_{j}}\right| & <\left|\frac{G\left(\theta_{j}\left(\boldsymbol{p}^{*}\right)\right)}{\left(1-p_{j}^{*}\right) G^{\prime}\left(\theta_{j}\left(\boldsymbol{p}^{*}\right)\right)}\right| \frac{\sum_{i \neq j} \frac{1}{\left(1-p_{i}^{* *}\right) G^{\prime}\left(\theta_{i}\left(\boldsymbol{p}^{* *}\right)\right)}}{\sum_{i=1 \frac{1}{\left(1-p_{i}^{*}\right) G^{\prime}\left(\theta_{i}\left(\boldsymbol{p}^{* *}\right)\right)}}^{s}} \\
/(\mathrm{A} 36) / & <\left|\frac{G\left(\theta_{j}\left(\boldsymbol{p}^{*}\right)\right)}{\left(1-p_{j}^{* *}\right) G^{\prime}\left(\theta_{j}\left(\boldsymbol{p}^{* *}\right)\right)}\right| \frac{\sum_{i \neq j} \frac{1}{\left(1-p_{i}^{* *}\right) G^{\prime}\left(\theta_{i}\left(\boldsymbol{p}^{* *}\right)\right)}}{\sum_{i=1}^{s} \frac{1}{\left(1-p_{i}^{* *}\right) G^{\prime}\left(\theta_{i}\left(\boldsymbol{p}^{* *}\right)\right)}} \\
/ G \text { decreasing/ } & <\left|\frac{G\left(\theta_{j}\left(\boldsymbol{p}^{* *}\right)\right)}{\left(1-p_{j}^{* *}\right) G^{\prime}\left(\theta_{j}\left(\boldsymbol{p}^{* *}\right)\right)}\right| \frac{\sum_{i \neq j} \frac{1}{\left(1-p_{i}^{* *}\right) G^{\prime}\left(\theta_{i}\left(\boldsymbol{p}^{* *}\right)\right)}}{\sum_{i=1}^{s} \frac{1}{\left(1-p_{i}^{* *}\right) G^{\prime}\left(\theta_{i}\left(\boldsymbol{p}^{* *}\right)\right)}}=\left|\frac{\partial \theta_{j}\left(\boldsymbol{p}^{* *}\right)}{\partial p_{j}}\right|,
\end{aligned}
$$

a contradiction. 


\section{A.6 Proof of Lemma 2}

Proof. It is left to the reader to differentiate $G(k)$ with respect to $k$ to show that $G(\cdot)$ is strictly decreasing. It is more work to demonstrate monotonicity of $H(\cdot)$. To do this, first notice that

$$
\begin{aligned}
\frac{d}{d x} \frac{1-e^{-x}}{1-e^{-x}(1+x)} & =\frac{e^{-x}}{1-e^{-x}(1+x)}-\frac{\left(1-e^{-x}\right) e^{-x} x}{\left[1-e^{-x}(1+x)\right]^{2}} \\
\frac{d}{d x}\left[1-e^{-x}\left(1+\frac{n}{(k+1)^{2}}\right)\right] & =e^{-x\left(1+\frac{n}{(k+1)^{2}}\right)}
\end{aligned}
$$

Hence,

$$
\begin{aligned}
& \frac{d}{d x}\left[\frac{1-e^{-x}}{1-e^{-x}(1+x)}\left[1-e^{-x}\left(1+\frac{n}{(k+1)^{2}}\right)\right]\right] \\
= & {\left[\frac{e^{-x}}{1-e^{-x}(1+x)}-\frac{\left(1-e^{-x}\right) e^{-x} x}{\left[1-e^{-x}(1+x)\right]^{2}}\right]\left[1-e^{-x}\left(1+\frac{n}{(k+1)^{2}}\right)\right] } \\
& +\frac{1-e^{-x}}{1-e^{-x}(1+x)} e^{-x}\left(1+\frac{n}{(k+1)^{2}}\right) \\
= & \frac{e^{-x}}{1-e^{-x}(1+x)}\left[1-\frac{\left(1-e^{-x}\right) x}{1-e^{-x}(1+x)}\right]\left[1-e^{-x}\left(1+\frac{n}{(k+1)^{2}}\right)\right] \\
= & +\frac{e^{-x}}{1-e^{-x}(1+x)}\left(1-e^{-x}\right)\left(1+\frac{n}{(k+1)^{2}}\right) \\
& +\underbrace{\frac{e^{-x}}{1-e^{-x}(1+x)} \underbrace{\frac{e^{-x}}{1-e^{-x}(1+x)}\left(1-e^{-x}\right)}\left(1+\frac{n}{(k+1)^{2}}\right) .}_{>0}\left[11-e^{-x}\left(1+\frac{n}{(k+1)^{2}}\right)\right]
\end{aligned}
$$

It follows that

$$
\begin{aligned}
& \frac{d}{d x}\left[\frac{1-e^{-x}}{1-e^{-x}(1+x)}\left[1-e^{-x}\left(1+\frac{n}{(k+1)^{2}}\right)\right]\right] \\
< & \frac{e^{-x}}{1-e^{-x}(1+x)}\left(\frac{1-e^{-x}-x}{1-e^{-x}(1+x)}\right)\left[1-e^{-x}\right]+\frac{e^{-x}}{1-e^{-x}(1+x)}\left(1-e^{-x}\right) \\
= & \frac{e^{-x}\left(1-e^{-x}\right)}{1-e^{-x}(1+x)}\left(\frac{1-e^{-x}-x}{1-e^{-x}(1+x)}+1\right) \\
= & \frac{e^{-x}\left(1-e^{-x}\right)}{\left[1-e^{-x}(1+x)\right]^{2}}\left(2\left(1-e^{-x}\right)-x\left(1+e^{-x}\right)\right) .
\end{aligned}
$$

Let $A(x)=2\left(1-e^{-x}\right)-x\left(1+e^{-x}\right)$ and note that $A(0)=2\left(1-e^{-0}\right)-0\left(1+e^{0}\right)=0$ and that

$$
A^{\prime}(x)=2 e^{-x}-\left(1+e^{-x}\right)+x e^{-x}=e^{-x}(1+x)-1<0 .
$$

We conclude that $A(x)=A(0)+\int_{0}^{x} A^{\prime}(z) d z<0$, implying that

$$
\frac{d}{d x}\left[\frac{1-e^{-x}}{1-e^{-x}(1+x)}\left[1-e^{-x}\left(1+\frac{n}{(k+1)^{2}}\right)\right]\right]<0 .
$$


In conclusion we have that for

$$
\begin{aligned}
H^{\prime}(k)= & \underbrace{\left.\frac{d}{d x}\right|_{x=\frac{n}{k+1}}\left[\frac{1-e^{-x}}{1-e^{-x}(1+x)}\left[1-e^{-x}\left(1+\frac{n}{(k+1)^{2}}\right)\right]\right]}_{<0}\left(-\frac{n}{(k+1)^{2}}\right) \\
& +\frac{1-e^{-x}}{1-e^{-x}(1+x)}\left[e^{-x}\left(1+\frac{2 n}{(k+1)^{3}}\right)\right]>0,
\end{aligned}
$$

which completes the proof. 